\title{
Regulation of calcium fluxes by GPX8, a type-II transmembrane peroxidase enriched at the mitochondria-associated endoplasmic reticulum membrane
}

Edgar Djaha Yoboue ${ }^{1 *}$, Alessandro Rimessi ${ }^{2 *}$, Tiziana Anelli ${ }^{1,3}$, Paolo Pinton ${ }^{2} \&$ Roberto Sitia $^{1,3}$

${ }^{1}$ Protein Transport and Secretion Unit, Division of Genetics and Cell Biology, IRCCS Ospedale San Raffaele

2 Dept. of Morphology, Surgery and Experimental Medicine, Section of Pathology, Oncology and Experimental Biology, Laboratory for Technologies of Advanced Therapies (LTTA), University of Ferrara, Ferrara, Italy.

${ }^{3}$ Università Vita-Salute San Raffaele, Milan, Italy.

* These authors contributed equally to this work.

\author{
Running title \\ Integrating Redox and $\mathrm{Ca}^{2+}$ Signaling @ MAM
}

Word count: 4591

References number: 44

Greyscale illustrations: 4

Color illustrations: 4 (online) 


\begin{abstract}
:
Glutathione peroxidases (GPXs) are enzymes present in almost all organisms with the primary function of limiting peroxides accumulation. In mammals, two of the eight members (GPX7 and GPX8) reside in the endoplasmic reticulum (ER). A peculiar feature of GPX8 is the concomitant presence of a conserved N-terminal transmembrane domain (TMD) and a Cterminal KDEL-like motif for ER localization.
\end{abstract}

Aims: Investigating whether and how GPX8 impacts $\mathrm{Ca}^{2+}$ homeostasis and signaling.

Results: We show that GPX8 is enriched in mitochondria-associated membranes (MAM) and regulates $\mathrm{Ca}^{2+}$ storage and fluxes. Its levels correlate with $\left[\mathrm{Ca}^{2+}\right]_{\mathrm{ER}}$, and cytosolic and mitochondrial $\mathrm{Ca}^{2+}$ fluxes. GPX7, which lacks a TMD, does not share these properties. Deleting or replacing the GPX8 TMD with an unrelated N-terminal membrane integration sequence abolishes all effects on $\mathrm{Ca}^{2+}$ fluxes, whilst appending the GPX8 TMD to GPX7 transfers the $\mathrm{Ca}^{2+}$ regulating properties.

Innovation and conclusion: The notion that the TMD of GPX8, in addition to its enzymatic activity, is essential for regulating $\mathrm{Ca}^{2+}$ dynamics, reveals a novel level of integration between redox related proteins and $\mathrm{Ca}^{2+}$ signaling/homeostasis. 


\section{Introduction}

The endoplasmic reticulum (ER) is a multifunctional organelle where about one third of the proteome folds and undergoes post-translational modifications, such as $\mathrm{N}$-glycosylation and disulfide bond formation. This network of tubules and cisternae is also involved in redox homeostasis, membrane lipid synthesis and $\mathrm{Ca}^{2+}$ storage and signaling $(8,18,20)$. The ER establishes close contacts with the plasma membrane and other organelles, such as mitochondria (28). In the last years, the so-called mitochondria associated membranes (MAM) emerged as key integration hubs for $\mathrm{Ca}^{2+}$, lipid metabolism and redox signaling homeostasis. MAM are subregions of the ER resulting from the closed contact between the ER and mitochondria. MAM are enriched in some $\mathrm{Ca}^{2+}$ handling proteins such as inositol 1,4,5-triphosphate receptors (IP3Rs), which release $\mathrm{Ca}^{2+}$ from the ER toward mitochondria and cytosol and for diverse physiological purposes $(13,15,32,35,42,43)$. Sarcoendoplasmic reticulum ATPase (SERCA) that guarantees prompt $\mathrm{ER} \mathrm{Ca}^{2+}$ replenishment is also in part located in MAM $(11,27)$. Moreover, it emerges that MAM also contain ER proteins primarily known for their involvement in protein folding and redox regulation $(3,24)$. Thus, we previously showed that Ero1 $\alpha$, a flavoprotein that generates $\mathrm{H}_{2} \mathrm{O}_{2}$ while promoting disulfide bond formation, is enriched in MAM and regulates the $\mathrm{ER} \mathrm{Ca}^{2+}$ storage and mitochondrial $\mathrm{Ca}^{2+}$ fluxes (1).

$\mathrm{H}_{2} \mathrm{O}_{2}$ is a double-edged sword for cells, acting as an essential second messenger but becoming harmful if in excess $(16,34)$. To limit its toxicity, $\mathrm{H}_{2} \mathrm{O}_{2}$ produced during Ero1dependent oxidative folding has to be cleared by luminal peroxidases (8). Glutathione peroxidase 8 (GPX8) is a type II transmembrane protein with rare structural features. Indeed, in addition to a highly conserved N-terminal transmembrane domain (TMD), GPX8 harbors a C-terminal KEDL ER localization motif $(23,39)$. GPX8 was shown to limit ER 
hyperoxidation and $\mathrm{H}_{2} \mathrm{O}_{2}$ leakage to the cytosol in conditions of deregulated Erol $\alpha$ activity (30). Furthermore, GPX8 is thought to form heterotrimeric complexes with Ero1 $\alpha$ and protein disulfide isomerase (PDI), thus coupling clearance of $\mathrm{H}_{2} \mathrm{O}_{2}$ with disulfide bond formation (31). Like Erol $\alpha$, GPX8 expression is induced by hypoxia (5). Considering the apparent synergies between Ero1 $\alpha$ and GPX8 and the $\mathrm{Ca}^{2+}$-regulatory roles of the former, we set out to investigate the involvement of GPX8 in $\mathrm{Ca}^{2+}$ homeostasis and signaling.

The experiments described here show that GPX8 is enriched in MAM and regulates $\mathrm{Ca}^{2+}$ signaling. We also demonstrate that its TMD is essential for the regulation of $\mathrm{Ca}^{2+}$ fluxes. Our data reveal a new player involved in the regulation of the physiological important $\mathrm{Ca}^{2+}$ dynamics and unveil the molecular features required for these regulatory functions. 


\section{Results \\ GPX8 is enriched in MAM in HeLa cells}

First, we assessed the subcellular localization of GPX8 by fractionation assay that allows the separation of ER, mitochondria and MAM (46). Analysis of the different fractions revealed that GPX8 is enriched in MAM (Figure 1), co-fractionating with other MAM markers such as IP3R3 and ASCL4. Instead, the chaperone GRP94 was mainly in the ER fraction. Thus, endogenous GPX8 accumulates in MAM in standard conditions.

\section{GPX8 level modulates $\mathrm{Ca}^{2+}$ dynamics}

Considering the strong enrichment of GPX8 in MAM, a key hub for $\mathrm{Ca}^{2+}$ signaling, we assessed the consequences of its overexpression (Figure 2A, supplementary figure 1A) or silencing (figure 2B, supplementary figure $1 \mathrm{~B}$ ) on intracellular $\mathrm{Ca}^{2+}$ dynamics. We first monitored the levels of $\mathrm{Ca}^{2+}$ in the ER $\left(\left[\mathrm{Ca}^{2+}\right]_{\mathrm{ER}}\right)$ and histamine-induced IP3-dependent release toward mitochondria and cytosol, which are crucial signaling processes. To this end, we used the well-known luminescent protein probe aequorin targeted to different subcellular compartments (4).

Cells overexpressing GPX8 displayed a significantly lower $\left[\mathrm{Ca}^{2+}\right]_{\mathrm{ER}}$ at steady-state (Figure 2A, left panel), as confirmed also in single-cell assay using the high $\mathrm{Ca}^{2+}$-sensitive FRETbased D1ER probe (Supplementary figure 2). Addition of the agonist histamine induces IP3 elevation, allowing the IP3R-dependent $\mathrm{Ca}^{2+}$-release from the ER toward the mitochondria and the cytosol. GPX8 overexpression strongly reduced these IP3-dependent $\mathrm{Ca}^{2+}$ fluxes (figure $2 \mathrm{~A}$, middle panels). The $\mathrm{ER} \mathrm{Ca}^{2+}$-release rate was also strongly reduced, suggesting that the lower mitochondrial and cytosolic responses were consequences of an impaired capacity of the ER to release $\mathrm{Ca}^{2+}$ in response to agonist stimulation. Using the high affinity $\mathrm{Ca}^{2+}$-probe FURA2-AM, we then investigated whether GPX8 level could alter the $\mathrm{Ca}^{2+}$ 
release induced by oxidative stimulus such as $\mathrm{H}_{2} \mathrm{O}_{2}$ that triggers a progressive $\mathrm{Ca}^{2+}$ release by IP3Rs independently of IP3 elevation (13). In cells overexpressing GPX8, the increase in $\left[\mathrm{Ca}^{2+}\right]_{\text {cyto }}$ evoked by $\mathrm{H}_{2} \mathrm{O}_{2}$ was also reduced (supplementary figure 3). GPX8 silencing had opposite effects, increasing $\mathrm{Ca}^{2+}$ fluxes into mitochondria and cytosol without significantly perturbing $\left[\mathrm{Ca}^{2+}\right]_{\mathrm{ER}}$ (Figure 2B). Thus, GPX8 plays a role in $\mathrm{Ca}^{2+}$ dynamics regulation.

\section{GPX8 level modulates both SERCA2b activity and ER $\mathrm{Ca}^{2+}$ leakage}

We focused next on the observation that GPX8 overexpression decreases the $\left[\mathrm{Ca}^{2+}\right]_{\mathrm{ER}}$ at steady-state. This parameter depends on the balance between ER refilling mediated mainly by SERCA and passive leak through IP3Rs or translocon components $(25,37,41,44)$. Thus, we used $\mathrm{Ca}^{2+}$ refilling assays to compare SERCA activity in cells overexpressing GPX8 and control cells. The activity of SERCA2b -the isoform expressed in HeLa cells- was slower in cells overexpressing GPX8 (Figure 3A, left panel). Moreover, passive $\mathrm{Ca}^{2+}$ efflux (i.e., leakage) from the ER was slightly increased by GPX8 overexpression, as determined by treatment with the SERCA inhibitor tBHQ (Figure 3A, right panel). Instead, GPX8 silencing increased the $\mathrm{ER} \mathrm{Ca}^{2+}$ entry rate (Figure 3B) without significant effects on $\mathrm{Ca}^{2+}$ leakage.

Thus, GPX8 levels modulate $\left[\mathrm{Ca}^{2+}\right]_{\mathrm{ER}}$ mainly through SERCA activity and partially by influencing passive $\mathrm{Ca}^{2+}$ leakage.

\section{The TMD of GPX8 is essential but not sufficient for regulating $\mathrm{Ca}^{2+}$ signaling}

Next, we asked whether the peculiar GPX8 topology was important for its activity in $\mathrm{Ca}^{2+}$ regulation. GPX8 is a type II membrane protein, with an 18 amino acids N-terminal cytosolic peptide, a hydrophobic stretch predicted to span the membrane (TMD) and the catalytic region in the ER lumen. The presence of a TMD is intriguing, considering that GPX8 has a Cterminal KEDL motif that mediates its retrieval to the ER (33). We generated a construct named S-GPX8, by substituting the GPX8 TMD with the cleavable ER signal peptide of the 
protein ERp44 (Figure 4A). Immunoblot and imaging assays confirmed that S-GPX8 is correctly expressed and localized in the ER (Figure 4B and C). Thus, the TMD is not mandatory for GPX8 ER retention. However, S-GPX8 had no effects on $\mathrm{Ca}^{2+}$ fluxes. Cells overexpressing it displayed a $\left[\mathrm{Ca}^{2+}\right]_{\mathrm{ER}}$ and mitochondrial and cytosolic $\mathrm{Ca}^{2+}$ responses similar to mock transfected cells (Figure 5A). In good accordance with our previous conclusions, overexpression of S-GPX8 had also no discernible effects on ER $\mathrm{Ca}^{2+}$ uptake or leakage (Figure 5B). Thus, the TMD is essential for the GPX8 effects on $\mathrm{Ca}^{2+}$ homeostasis.

To determine the intrinsic role of the GPX8 TMD, we fused the GPX8 N-terminus to an irrelevant protein. To this end, we chose the Halotag (Figure 6A, B), a recombinant protein capable of binding covalently fluorescent ligands and shown not to alter the properties of other ER proteins $(19,22)$. Neither TMD8-Halo nor a variant with a cleavable leader sequence (S-Halo) affected $\left[\mathrm{Ca}^{2+}\right]_{\mathrm{ER}}$ and release (Figure 6C, D). Altogether, these data suggest that while the TMD of GPX8 is essential for its effects on $\mathrm{Ca}^{2+}$ dynamics, element(s) present in the luminal portion is (are) also required for full regulation.

\section{Regulation of $\mathrm{Ca}^{2+}$ dynamics relies on both the TMD and peroxidatic cysteines of GPX8.}

To determine whether the peroxidatic activity of GPX8 could be required for $\mathrm{Ca}^{2+}$ regulation, we analyzed the effects on $\mathrm{Ca}^{2+}$ signaling of a GPX8 mutant in which cysteines 79 and 108 -essential for enzymatic activity (31,45)- had been replaced with serine and alanine, respectively (GPX8-CCSA). When expressed at levels similar to WT-GPX8, the GPX8CCSA mutant lost most of its inhibitory effect on $\left[\mathrm{Ca}^{2+}\right]_{\mathrm{ER}}$ and mitochondrial $\mathrm{Ca}^{2+}$ responses (Figures 6E,F), suggesting that the GPX8 peroxidatic cysteines are also necessary to modulate efficiently $\mathrm{Ca}^{2+}$ homeostasis.

These notions were strengthened by performing a comparative analysis with GPX7 (figure 7). Like GPX8, GPX7 resides in the ER and both enzymes share significant structural 
similarities excepting that GPX7 harbors a cleavable ER signal peptide and hence behaves as a soluble ER protein $(23,29,40)$. GPX7 overexpression had no obvious effects on $\mathrm{Ca}^{2+}$ dynamics (figure 7D). However, a fusion protein in which the GPX7 N-terminus is substituted with the GPX8 TMD displayed similar properties to GPX8, with a reduction of $\left[\mathrm{Ca}^{2+}\right]_{\mathrm{ER}}$ and IP3R-dependent mitochondrial and cytosolic $\mathrm{Ca}^{2+}$ responses (Figure 7). Altogether these results indicate that an active GPX moiety needs to be bound to the membrane in order to control ER $\mathrm{Ca}^{2+}$ homeostasis.

The transmembrane domain of GPX8 is specifically required for regulation of $\mathrm{Ca}^{2+}$ dynamics

Next, we sought evidence to determine whether membrane insertion per se was sufficient for affecting $\mathrm{Ca}^{2+}$ dynamics, or wether information specifically contained within the conserved GPX8 TMD sequence was required. To address this question, we replaced the entire GPX8 N-terminal region or the sole TMD with the corresponding portions of asialoglycoprotein receptor 1 (ASGR1), a type II membrane protein involved in serum glycoprotein turnover $(10,38)$ (Figure 8 A). Clearly, both ASGR-GPX8 chimeric molecules (CA-TMDA-GPX8 and C8-TMDA-GPX8) were properly expressed and ER localized, reconfirming that the GPX8-N terminal region is not necessary for ER localization (Figure 8B, C). However, cells overexpressing CA-TMDA-GPX8 or C8-TMDA-GPX8 showed no alteration of $\left[\mathrm{Ca}^{2+}\right]_{\mathrm{ER}}$ and displayed IP3-dependent mitochondrial responses similar to mocktransfectants (Figure 8D). Thus, the GPX8 transmembrane stretch sequence is specifically needed for the regulation of $\mathrm{Ca}^{2+}$ dynamics by GPX8. 


\section{Discussion}

Glutathione peroxidases are found in almost all kingdoms of life, playing important roles in fertility, inflammation, cancer (6,39). Two of the eight mammalian family members, GPX7 and GPX8, are localized in the ER where they scavenge $\mathrm{H}_{2} \mathrm{O}_{2}$ produced by Erol $\alpha$, avoiding lethal oxidative stress and further fueling disulfide bond formation via PDI or other resident oxidoreductases $(9,23,29,45)$. In this study, we focused on GPX8, which is the last identified family member, presenting a peculiar topology as a type II membrane protein with a KEDL C-terminal sequence and still poorly studied in vivo.

The first conclusion that emerges from our studies is that GPX8 is enriched in MAM (Figure 1). This result is strengthened by independent studies showing that the NS3-4A protease of the hepatitis C virus targets MAM in human cells (17), with GPX8 being the tophit target of the protease (21). Thus, GPX8 is a novel MAM-enriched redox protein.

We also showed that GPX8 level affects $\mathrm{Ca}^{2+}$ homeostasis and signaling. Cells overexpressing GPX8 have reduced $\left[\mathrm{Ca}^{2+}\right]_{\mathrm{ER}}$ and IP3-dependent mitochondrial and cytosolic $\mathrm{Ca}^{2+}$ transients, while its silencing had opposite effects (Figure 2).

A third important conclusion of our work is that the TMD of GPX8 is essential for impacting $\mathrm{Ca}^{2+}$ homeostasis (Figure 5). Interestingly, regulatory information is specifically contained in the GPX8 transmembrane sequence and not in the topology that it warrants, since the ASGR1 TMD could not be a surrogate (Figure 8). In addition, overexpressing GPX7 had no effects, unless equipped with the GPX8 TMD (Figure 7). The inactivity of GPX7, S-GPX8 and chimeric TMD8-Halo proteins excludes the possibilities that the impaired $\mathrm{Ca}^{2+}$ dynamics were mere consequences of overexpressing redox related proteins. Thus, for the first time, relevance for the GPX8 TMD is revealed. These results were obtained in different cell types (supplementary figure 4) and also in condition of oxidative stimulus (supplementary figure 3), 
suggesting a general $\mathrm{Ca}^{2+}$ signaling control process and underscoring the peculiar properties of GPX8.

What could be so special about the GPX8 TMD? We first considered that the GPX8 TMD could be essential for MAM targeting. However, soluble GPX8 is also enriched in the MAM (Supplementary figure 5) while being inactive on $\mathrm{Ca}^{2+}$ fluxes (figure 5). Thus, being enriched in MAM is not sufficient and an active GPX moiety must contain a peculiar aminoacid sequence allowing its membrane insertion in order to affect $\mathrm{Ca}^{2+}$ signaling. The TMD might anchor GPX8 in a specialized place, where its level could regulate the $\mathrm{Ca}^{2+}$ machinery. In this regard, our results showing an impairment of the ${\mathrm{ER} \mathrm{Ca}^{2+}}^{2}$ refilling (figure 3 and 5) without basal mitochondrial $\mathrm{Ca}^{2+}$ changes (supplementary figure 6), point at SERCA as a possible target of GPX8. GPX8 overexpression does not influence the levels of SERCA or other $\mathrm{Ca}^{2+}$ related channels (supplementary figure 7). But, co-immunoprecipitation experiments revealed associations between GPX8 and SERCA. However, this interaction also occurs without the GPX8 TMD (supplementary figure 8). These results strengthen the notion of interplay between GPX8 and SERCA but also suggest more complex mechanism(s). For example, SERCA regulation could be indirect, and involve other molecule(s) recruited by the GPX8 TMD. Beside the latter's identities, other questions such as how GPX8 or other ER redox proteins are localized to the MAM will be of interest $(3,12)$.

In sum, our data identify GPX8 as a novel component of the MAM signaling hub, underlying for the first time, the essential role of its TMD in influencing integration of redox and $\mathrm{Ca}^{2+}$ signaling processes. 


\section{Innovation (100 words max)}

The interplays between redox and $\mathrm{Ca}^{2+}$ homeostasis/signaling and the dynamics of interorganellar communication are of fundamental importance in cell pathophysiology. The involvement of the ER peroxidases in these expanding research fields was still unexplored. Our study identifies GPX8 as a novel important integrator of redox and $\mathrm{Ca}^{2+}$ signals at the mitochondria-endoplasmic reticulum interface and uncovers a critical role for its conserved transmembrane domain in the regulation of $\mathrm{Ca}^{2+}$ dynamics. 


\section{Materials and methods}

\section{Reagents, cell lines and transfection procedures}

Polyclonal anti-GPx8 antibody was a kind gift of D. Moradpour (Lausanne, $\mathrm{CH}$ ) and is available from Adipogen (AG-25B-0028-C100). Polyclonal anti-PDI was a kind gift of I. Braakman (Utrecht, NL). Other antibodies used for the study are anti-Ero1 $\alpha 2 \mathrm{G} 4$ (36), antiHalo tag (G9281, Promega), anti-ASCL4, anti-TOM20 and anti-HSP60 (sc-47997, sc-11415 and sc-13115 respectively, Santa Cruz Biotechnology), anti-SERCA2 (4388S, Cell Signaling), anti-IP3R1 (AB5804, Abcam) anti-IP3R3 (610312, BD Transduction Laboratories), anti-MCU and anti-Actin (HPA016480 and A1978 respectively, Sigma Aldrich), anti-Calnexin and anti-GRP94 (SPA-860 and SPA-851 respectively, Enzo Life Sciences). Fluorescent goat anti-mouse and anti-rabbit IgG $(\mathrm{H}+\mathrm{L})$ Alexa Fluor conjugated 647, 546 and 488 were from Invitrogen Molecular Probes (Eugene, Oregon, USA). Culture media were from Invitrogen life technologies. HeLa cell lines were purchased from ATCC. Tissue culture, transfection, and silencing were performed as described previously (2). All the other reagents were from Sigma-Aldrich (St. Louis, MO).

\section{Subcellular fractionation}

After homogenization of the cells, crude mitochondrial and microsomal fractions were separated by classical differential centrifugation. Subsequently, the crude mitochondrial fraction was resuspended in isolation buffer $(250 \mathrm{mM}$ mannitol, $5 \mathrm{mM}$ Hepes, $0.5 \mathrm{mM}$ EGTA, pH 7.4) and further separated on a $30 \%$ Percoll gradient to obtain low-density (denoted as MAM) and high-density (denoted as pure mitochondria, Mito Pure) fractions as described in (46). Aliquots of the fractions were collected and analyzed by western-blot after SDS-PAGE performed on equal quantity of proteins.

Proteins extraction, electrophoresis, and western-blot 
Cells were trypsinized, washed in PBS and lysed in RIPA buffer (150 mM NaCl, $1 \% \mathrm{NP}$ 40, $0.1 \%$ SDS, $5 \mathrm{mM}$ Tris- $\mathrm{HCl} \mathrm{pH}$ 8.0) supplemented with NEM $10 \mathrm{mM}$ and protease inhibitors cocktail (Roche). After centrifugation at $14,000 \mathrm{~g}$ at $4^{\circ} \mathrm{C}$, proteins were separated by reducing SDS-PAGE on gradient 4-12\% Bis-Tris polyacrylamide precast gel gradient (Life Technologies) or $15 \%$ homemade Tris-glycine SDS-PAGE.

After electrotransfer onto nitrocellulose membrane and probed with the desired antibodies, the proteins were visualized with FLA-900 Starion (when fluorescent secondary antibodies have been used) or electrochemiluminescence (ECL prime, GE. Healthcare) when using HRP coupled secondary antibodies.

\section{Calcium measurements}

Cells were plated onto $13 \times 13 \mathrm{~mm}$ coverslips at a density of $5 \times 10^{5}$ cells/coverslip, then cotransfected with organelle-specific aequorin-based probes and GPX8 or its mutants The probes employed (cytAEQ, mtAEQ and erAEQ) are chimeric aequorins targeted to the cytosol, mitochondria and ER, respectively (4). For the experiments with cytAEQ and mtAEQ, cells were incubated with $5 \mu \mathrm{M}$ coelenterazine for 1-2 hours in DMEM supplemented with $1 \%$ FCS. Then the coverslip with transfected cells was placed in a perfused chamber with thermostat, located in close proximity of a low noise photomultiplier, with a built-in amplifier-discriminator. To reconstitute the erAEQ with high efficiency, the luminal $\left[\mathrm{Ca}^{2+}\right]$ of the stores first had to be reduced. This was achieved by incubating the cells for 45 minutes at $4^{\circ} \mathrm{C}$ in Krebs Ringer Buffer (KRB), supplemented with $5 \mu \mathrm{M}$ coelenterazine, the $\mathrm{Ca}^{2+}$ ionophore ionomycin, and $600 \mu \mathrm{M}$ EGTA. After 45 min, cells were extensively washed with $\mathrm{KRB}$, supplemented with $2 \%$ bovine serum albumin, and then transferred to the perfusion chamber. All aequorin measurements were carried out in KRB supplemented with either $1 \mathrm{mM} \mathrm{CaCl}_{2}$ (cytAEQ and mtAEQ) or $100 \mu \mathrm{M}$ EGTA (erAEQ). Agonists (100 $\mu \mathrm{M}$ histamine, $1 \mu \mathrm{M}$ Bradykinin) were perfused to the same medium to induce 
IP3-dependent $\mathrm{Ca}^{2+}$ release from ER, generating rapid rise in cytosolic and mitochondrial [Ca2+] followed by a gradual decline to a lower plateau, as reported in the supplementary figure 1 . The experiments were terminated by lysing cells with $100 \mu \mathrm{M}$ Triton in a hypotonic $\mathrm{Ca}^{2+}$-containing solution $\left(10 \mathrm{mM} \mathrm{CaCl}{ }_{2}\right.$ in $\left.\mathrm{H}_{2} \mathrm{O}\right)$, thus discharging the remaining aequorin pool. The output of the discriminator was captured by a Thorn-EMI photon counting board and stored in an IBM-compatible computer for further analyses. The aequorin luminescence data were calibrated off-line into $\left[\mathrm{Ca}^{2+}\right]$ values, using a computer algorithm based on the $\mathrm{Ca}^{2+}$ response curve of wt or mutated aequorin (4). Statistical data are expressed as mean of agonist-dependent ER-, mitochondrial- and cytosolic $\mathrm{Ca}^{2+}$ response \pm S.E.

To measure SERCA2b activity, the $\mathrm{Ca}^{2+}$ uptake rate was analysed by OriginLab analysis program, measured from the first derivative considering the first 30 seconds of measurements from $\mathrm{Ca}^{2+}$ addition in erAEQ-based experiments. The data obtained are expressed as mean of $\mathrm{ER} \mathrm{Ca}^{2+}$ accumulation rate per second \pm S.E. $(\mu \mathrm{M} / \mathrm{s})$. For measurements of ER $\mathrm{Ca}^{2+}$ leak rate, HeLa cells were co-transfected with erAEQ and the indicated constructs or siRNA. 36h after transfection, depletion of $\mathrm{Ca}^{2+}$ stores and AEQ reconstitution was performed. The coverslip with the cells was transferred to the luminometer and perfused with $\mathrm{KRB} / \mathrm{Ca}^{2+}$ until the steady-state $\left[\mathrm{Ca}^{2+}\right]_{\mathrm{ER}}$ was reached. The ER was refilled by exposing cells to extracellular $\left[\mathrm{Ca}^{2+}\right]$ ranging from 0.1 to $1 \mathrm{mM}$, in order to obtain different levels of steady state $\left[\mathrm{Ca}^{2+}\right]_{\mathrm{ER}}$. Cells were then treated with the SERCA inhibitor tBHQ $(30 \mu \mathrm{M})$ and the consequent decrease of $\left[\mathrm{Ca}^{2+}\right]_{\mathrm{ER}}$, due to passive efflux, was then analysed. The maximal rates of $\mathrm{Ca}^{2+}$ release at different $\left[\mathrm{Ca}^{2+}\right]_{\mathrm{ER}}$ values were calculated (measured from the first derivative considering $15 \mathrm{~s}$ after tBHQ addition). To minimize the effects of the $\left[\mathrm{Ca}^{2+}\right]_{\mathrm{ER}}$ on passive efflux, cells in the 250-350 $\mu \mathrm{M}$ range of plateau values were compared. The rate of decline of $\left[\mathrm{Ca}^{2+}\right]_{\mathrm{ER}}$ must reflect the rate of $\mathrm{ER} \mathrm{Ca}^{2+}$ extrusion, shown as response mean \pm S.E. $(\mu \mathrm{M} / \mathrm{s})$ for each experimental conditions. 


\section{Plasmids construction}

For generating the plasmid overexpressing a HA-tagged version of the wild-type form of GPX8 (GPX8 WT) and the catalytically inactive GPx8 (GPx8-C79S), GPx8-HA sequences on the pRK7 vector (pKEHS768; a kind gift of L. Ruddock, Oulu, Finland) and on the pcDNA 3.0 vector (kindly provided by C. Appenzeller-Herzog, Basel, Switzerland) respectively, were excised and subcloned into pcDNA3.1 using BamHI and HindIII. The plasmid expressing GPX8-C79S was used as template for site-directed mutagenesis by PCR of C108 in A in order to generate the plasmid GPX8-CCSA To generate the transmembraneless form of GPX8 (S-GPX8), the sequence encoding for the HA-tagged luminal part of GPX8 was amplified by PCR using the following primers: GGCGCgcgatcgctgaaAAACCTAAAATCAACAGC; CCGGggatccTCATAGATCC TCTTTCTTtgeg and the plasmid pKEHS768 as template. The PCR product was digested with SgfI and BamHI restriction enzymes and cloned in frame with the encoding sequence of the signal peptide of ERp44 into a pcDNA3.1 vector. The S-Halo encoding plasmid has been previously described (22). For generating TMD8-Halo, the primers ggtcgactctagaccaccATGGAGCCTC and ccetgcgatcgcTTTAGGTTTgaggaa were used for amplifying by PCR the residues 1-43 of GPX8 using the plasmid pKEHS768 as template. The PCR product and the S-Halo plasmid were digested with the SgfI and XbaI enzymes and ligated for completing the construction. HA-tag and the KREDL motif naturally found at the C-terminus of GPX7 were added through PCR to generate the plasmid overexpressing GPX7. This plasmid was used as template for amplifying by PCR the sequence encoding for the matured form of GPX7. The PCR product was substituted for the HALO tag in the plasmid C8-TMD8-Halo for generating the C8-TMD8-GPX7 encoding plasmid. GPX8 small interfering RNA was purchased from Ambion (Life Technologies). For obtaining the plasmid encoding for GPX8 fused to the Transmembrane domain of ASGR1 (CA-TMDA-GPX8), the 
sequence encoding for the 67 first N-terminus residues of ASGR1 was amplified by PCR. The PCR product was substituted for the signal peptide of ERp44 present in the S-GPX8 plasmid. The plasmid CA-TMDA-GPX8 was used as template for PCR generating the plasmid C8TMDA-GPX8. Primer sequences are available through request. All constructs were validated by sequencing (GATC Biotech, Germany). Silencing was performed using RNAiMAX (Invitrogen, Carlsbad, California) based of manufacturer instructions.

\section{Fluorescence microscopy experiments}

Glass-plated cells were transfected with the plasmid of interest and grown for 48 hours. Cells were then fixed using $4 \%$ paraformaldehyde, permeabilized with $0.2 \%$ Triton X-100 and then incubated with the suitable antibodies (1:100 and 1:200 dilutions for the primary and secondary respectively). After mounting, images were acquired and analyzed using DeltaVision RT Deconvolution System (GE Healthcare)

\section{Measurements of ER $\mathrm{Ca}^{2+}$ dynamics with DIER}

Intraluminal $\mathrm{Ca}^{2+}$ dynamics were measured using single-cell $\mathrm{Ca}^{2+}$ imaging with the $\mathrm{Ca}^{2+}$ sensitive FRET (fluorescence resonance energy transfer)-based chameleon protein D1ER (26). HeLa cells were co-transfected with D1ER and GPX8 WT. After 36 hours, coverslips were placed in $1 \mathrm{ml}$ of $\mathrm{KRB} / \mathrm{EGTA} \mathrm{Ca}{ }^{2+}$ free and TBHQ $20 \mu \mathrm{M}$ for $15 \mathrm{~min}$. After wash the coverslips were maintained in $\mathrm{KBR}^{2+}$ free and images were captured with METAFLUOR 7.0 Software (Universal Imaging) at $\lambda_{\text {excitation }}=430 \mathrm{~nm}$ and $\lambda_{\text {emission }}=470$ and $535 \mathrm{~nm}$ every 1 second using a Zeiss Axiovert 200M inverted microscope equipped with a C-Apochromat 40x/1.2 W CORR objective and a cooled CCD camera (Photometrics). CFP emission and yellow fluorescent protein (YFP) FRET emission were alternately collected at 470 and 535 $\mathrm{nm}$, respectively. The FRET signal was normalized to the CFP emission intensity, and changes in $\mathrm{ER} \mathrm{Ca}^{2+}$ were expressed as the ratio of the emissions at 535 and $470 \mathrm{~nm}$. Cells 
were perfused with $\mathrm{KRB} \mathrm{Ca}^{2+}$ free at the beginning of the experiment and the baseline of the FRET ratio was measured, which corresponds to ER depleted $\mathrm{Ca}^{2+}$ level. Then, $\mathrm{Ca}^{2+}(1 \mathrm{mM})$ was added to the perfusion, thus stimulating $\mathrm{ER} \mathrm{Ca}^{2+}$ refilling.

\section{Fura-2/AM measurements}

The cytosolic free $\mathrm{Ca}^{2+}$ concentration $\left(\left[\mathrm{Ca}^{2+}\right]_{\mathrm{c}}\right)$ was evaluated using the fluorescent $\mathrm{Ca}^{2+}$ indicator Fura-2 acetoxymethyl ester (Fura-2/AM; Molecular Probes). Briefly, cells were incubated in medium supplemented with $2.5 \mu \mathrm{M}$ Fura-2/AM for $30 \mathrm{~min}$, washed with KRB buffer to remove the extracellular probe, supplied with preheated KRB buffer (supplemented with $1 \mathrm{mM} \mathrm{CaCl} 2)$, and placed in a thermostated $\left(37^{\circ} \mathrm{C}\right)$ incubation chamber on the stage of an inverted fluorescence microscope (Zeiss Axiovert 200). Dynamic video imaging was performed using the Metafluor software (Universal Imaging Corporation, USA). Fluorescence was measured every $100 \mathrm{~ms}$ with the excitation wavelength alternating between 340 and 380 $\mathrm{nm}$ and the emission fluorescence being recorded at $510 \mathrm{~nm}$. At the end of the experiment, a region free of cells was selected, and one averaged background frame was collected at each excitation wavelength for background correction. The $\left[\mathrm{Ca}^{2+}\right]_{c}$ was calculated by the ratio method using the equation: $\left[\mathrm{Ca}^{2+}\right]_{\mathrm{c}}=\mathrm{Kd}(\mathrm{R}-\mathrm{Rmin}) /(\mathrm{R}-\mathrm{Rmax}) \mathrm{x} \mathrm{Sf} / \mathrm{Sf} 1$ where $\mathrm{Kd}$ is dissociation constant of Fura-2/AM for $\left(\mathrm{Ca}^{2+}\right)$ taken as $240 \mathrm{nM}$ at $37{ }^{\circ} \mathrm{C}$, $\mathrm{R}$ is ratio of fluorescence for Fura-2/AM at the two excitation wavelengths, F340/F380, Rmax is ratio of fluorescence in the presence of excess of $\mathrm{Ca}^{2+}$ obtained by lysing the cells with $10 \mu \mathrm{M}$ ionomycin (Sigma Aldrich), Rmin is ratio of fluorescence in the presence of minimal $\mathrm{Ca}^{2+}$ obtained by lysing the cells and then chelating all the $\mathrm{Ca}^{2+}$ with $0.5 \mathrm{M}$ EGTA, Sf2 is fluorescence of $\mathrm{Ca}^{2+}$ free form of Fura-2/AM at 380nm excitation wavelength and Sf1 is fluorescence of $\mathrm{Ca}^{2+}$ bound form of Fura-2/AM at $380 \mathrm{~nm}$ excitation wavelength.

\section{Mitochondrial $\mathrm{Ca}^{2+}$ concentration measurements with 2mt-GCaMP6m}


To test resting mitochondrial $\mathrm{Ca}^{2+}$ concentrations with high sensitivity, we used the $\mathrm{Ca}^{2+}$ probe based on the last-generation GCaMP probe targeted to the mitochondrial matrix (7). We chose the GCaMP6m version because it had the highest $\mathrm{Ca}^{2+}$ affinity. To measure the signal independent of variations in basal fluorescence intensity due to the variable expression levels of the probe, we took advantage of the isosbestic point in the GCaMP6m excitation spectrum; exciting GCaMP6m at $410 \mathrm{~nm}$ led to fluorescence emission that was not $\mathrm{Ca}^{2+}$ dependent. As a consequence, the ratio between the excitation wavelengths of 474 and $410 \mathrm{~nm}$ was proportional to the $\mathrm{Ca}^{2+}$ concentration and independent of probe expression levels. Cells were imaged with an IX-81 automated epifluorescence microscope (Olympus) equipped with a $40 \times$ oil immersion objective and an ORCA-R2 charge-coupled device camera (Hamamatsu Photonics). 


\section{Acknowledgments}

We thank members of the Pinton, Sitia and van Anken laboratories for helpful suggestions and discussions, Claudio Fagioli and Roberta Colzani for precious technical and secretarial assistance, respectively. We are extremely grateful to Drs. Christian Appenzeller-Herzog, Lloyd Ruddock and Lei Wang for generous gifts of plasmids and suggestions. We thank Dr. Julia Birk, Dr. Darius Moradpour and Dr. Ineke Braakman for providing useful reagents. ED Y. thanks Sara Alameddine, Andrea Giordano, Lucilla Sciaqua and Paolo Tesauro for help during plasmid constructions. P.P. is grateful to Camilla degli Scrovegni for her continuous support. Part of this work was carried out in the Advanced Light and Electron Microscopy BioImaging Center (ALEMBIC) of San Raffaele Scientific Institute and Vita-Salute University. This work was supported in part by grants from (AIRC (IG14559), Fondazione Cariplo, Fondazione Banca del Monte di Lombardia Ministero della Salute (PE-201102352286), and Telethon (GGP15059) to R.S. ED Y. was recipient of a Marie Curie Fellowship through the INVEST-COFUND program (PCOFUND-GA-2010-267264 INVEST). This work was also supported by the local funds from the University of Ferrara to A.R. and P.P. and grants from the Italian Ministry of Health (GR-2011-02346964), the Italian Cystic Fibrosis Foundation (FFC \# 20/2015) to A.R, and by grants from the Italian Association for Cancer Research (AIRC, IG-18624), Telethon (GGP15219B), the Italian Cystic Fibrosis Foundation (FFC \# 19/2014), the Italian Ministry of Education, University and Research (COFIN: 20129JLHSY_002, FIRB: RBAP11FXBC_002, Futuro in Ricerca: RBFR10EGVP_001) and the Italian Ministry of Health to P.P.

\section{Author disclosure statement}

No competing financial interests exist. 


\section{List of abbreviations}

ACSL4= Long-chain-fatty-acid--CoA ligase 4

$\mathrm{ER}=$ Endoplasmic reticulum

ERO1= endoplasmic reticulum oxidoreductin 1

GPX= Glutathione peroxidase

IP3= Inositol 1,4,5-trisphosphate

IP3R = Inositol 1,4,5-trisphosphate receptor

$\mathrm{KRB}=$ Krebs ringer buffer

MAM= Mitochondria associated membranes

MCU $=$ Mitochondrial calcium uniporter

$\mathrm{PDI}=$ Protein disulfide isomerase

SERCA $=$ Sarcoendoplasmic reticulum calcium ATPase

TOM20 $=$ Mitochondrial import receptor subunit TOM20 homolog

$\mathrm{TMD}=$ Transmembrane domain

TMD8 $=$ Transmembrane domain of GPX8

$\mathrm{tBHQ}=$ 2,5-di-(terbutyl)-1,4-benzohydroquinone 


\section{References}

1. Anelli T, Bergamelli L, Margittai E, Rimessi A, Fagioli C, Malgaroli A, Pinton P, Ripamonti M, Rizzuto R, and Sitia R: Ero1 $\alpha$ Regulates Ca2+ Fluxes at the Endoplasmic Reticulum-Mitochondria Interface (MAM). Antioxid Redox Signal 16: 1077-1087, 2012.

2. Anelli T, Ceppi S, Bergamelli L, Cortini M, Masciarelli S, Valetti C, and Sitia R: Sequential steps and checkpoints in the early exocytic compartment during secretory IgM biogenesis. EMBO J 26: 4177-4188, 2007.

3. Appenzeller-Herzog C and Simmen T: ER-luminal thiol/selenol-mediated regulation of Ca2+ signalling. Biochem Soc Trans 44: 452-459, 2016.

4. Bonora M, Giorgi C, Bononi A, Marchi S, Patergnani S, Rimessi A, Rizzuto R, and Pinton P: Subcellular calcium measurements in mammalian cells using jellyfish photoprotein aequorin-based probes. Nat Protoc 8: 2105-2118, 2013.

5. Bosello-Travain V, Forman HJ, Roveri A, Toppo S, Ursini F, Venerando R, Warnecke $\mathrm{C}$, Zaccarin M, and Maiorino M: Glutathione peroxidase 8 is transcriptionally regulated by HIF $\alpha$ and modulates growth factor signaling in HeLa cells. Free Radic Biol Med 81: $58-68,2015$.

6. Brigelius-Flohé R and Maiorino M: Glutathione peroxidases. Biochim Biophys Acta BBA - Gen Subj 1830: 3289-3303, 2013.

7. Chen T-W, Wardill TJ, Sun Y, Pulver SR, Renninger SL, Baohan A, Schreiter ER, Kerr RA, Orger MB, Jayaraman V, Looger LL, Svoboda K, and Kim DS: Ultrasensitive fluorescent proteins for imaging neuronal activity. Nature 499: 295-300, 2013.

8. Csala M, Kereszturi E, Mandl J, and Bánhegyi G: The Endoplasmic Reticulum As the Extracellular Space Inside the Cell: Role in Protein Folding and Glycosylation. Antioxid Redox Signal, 2012.

9. Delaunay-Moisan A and Appenzeller-Herzog C: The antioxidant machinery of the endoplasmic reticulum: Protection and signaling. Free Radic Biol Med 83: 341-351, 2015.

10. G Ashwell and Harford and J: Carbohydrate-Specific Receptors of the Liver. Annu Rev Biochem 51: 531-554, 1982. 
11. García-Pérez C, Hajnóczky G, and Csordás G: Physical Coupling Supports the Local $\mathrm{Ca} 2+$ Transfer between Sarcoplasmic Reticulum Subdomains and the Mitochondria in Heart Muscle. J Biol Chem 283: 32771-32780, 2008.

12. Gilady SY, Bui M, Lynes EM, Benson MD, Watts R, Vance JE, and Simmen T: Ero1 $\alpha$ requires oxidizing and normoxic conditions to localize to the mitochondria-associated membrane (MAM). Cell Stress Chaperones 15: 619-629, 2010.

13. Giorgi C, Missiroli S, Patergnani S, Duszynski J, Wieckowski MR, and Pinton P: Mitochondria-Associated Membranes: Composition, Molecular Mechanisms, and Physiopathological Implications. Antioxid Redox Signal 22: 995-1019, 2015.

14. Grupe M, Myers G, Penner R, and Fleig A: Activation of store-operated I(CRAC) by hydrogen peroxide. Cell Calcium 48: 1-9, 2010.

15. Hajnóczky G, Csordás G, Madesh M, and Pacher P: Control of apoptosis by IP3and ryanodine receptor driven calcium signals. Cell Calcium 28: 349-363, 2000.

16. Holmström KM and Finkel T: Cellular mechanisms and physiological consequences of redox-dependent signalling. Nat Rev Mol Cell Biol 15: 411-421, 2014.

17. Horner SM, Liu HM, Park HS, Briley J, and Gale M: Mitochondrial-associated endoplasmic reticulum membranes (MAM) form innate immune synapses and are targeted by hepatitis C virus. Proc Natl Acad Sci 108: 14590-14595, 2011.

18. Kakihana T, Nagata K, and Sitia R: Peroxides and peroxidases in the endoplasmic reticulum: integrating redox homeostasis and oxidative folding. Antioxid Redox Signal 16: 763-771, 2012.

19. Los GV, Encell LP, McDougall MG, Hartzell DD, Karassina N, Zimprich C, Wood MG, Learish R, Ohana RF, Urh M, Simpson D, Mendez J, Zimmerman K, Otto P, Vidugiris G, Zhu J, Darzins A, Klaubert DH, Bulleit RF, and Wood KV: HaloTag: A Novel Protein Labeling Technology for Cell Imaging and Protein Analysis. ACS Chem Biol 3: 373-382, 2008.

20. Lynes EM and Simmen T: Urban planning of the endoplasmic reticulum (ER): How diverse mechanisms segregate the many functions of the ER. Biochim Biophys Acta BBA - Mol Cell Res 1813: 1893-1905, 2011.

21. Morikawa K, Gouttenoire J, Hernandez C, Dao Thi VL, Tran HTL, Lange CM, Dill MT, Heim MH, Donzé O, Penin F, Quadroni M, and Moradpour D: Quantitative proteomics 
identifies the membrane-associated peroxidase GPx 8 as a cellular substrate of the hepatitis C virus NS3-4A protease. Hepatology 59: 423-433, 2014.

22. Mossuto MF, Sannino S, Mazza D, Fagioli C, Vitale M, Yoboue ED, Sitia R, and Anelli T: A Dynamic Study of Protein Secretion and Aggregation in the Secretory Pathway. PLoS ONE 9: e108496, 2014.

23. Nguyen VD, Saaranen MJ, Karala A-R, Lappi A-K, Wang L, Raykhel IB, Alanen HI, Salo KEH, Wang C, and Ruddock LW: Two Endoplasmic Reticulum PDI Peroxidases Increase the Efficiency of the Use of Peroxide during Disulfide Bond Formation. $J$ Mol Biol 406: 503-515, 2011.

24. Nunes P and Demaurex N: Redox Regulation of Store-Operated Ca2+ Entry. Antioxid Redox Signal 21: 915-932, 2013.

25. Oakes SA, Scorrano L, Opferman JT, Bassik MC, Nishino M, Pozzan T, and Korsmeyer SJ: Proapoptotic BAX and BAK regulate the type 1 inositol trisphosphate receptor and calcium leak from the endoplasmic reticulum. Proc Natl Acad Sci U S A 102: 105-110, 2005.

26. Palmer AE, Jin C, Reed JC, and Tsien RY: Bcl-2-mediated alterations in endoplasmic reticulum $\mathrm{Ca} 2+$ analyzed with an improved genetically encoded fluorescent sensor. Proc Natl Acad Sci U S A 101: 17404-17409, 2004.

27. Patergnani S, Suski JM, Agnoletto C, Bononi A, Bonora M, De Marchi E, Giorgi C, Marchi S, Missiroli S, Poletti F, Rimessi A, Duszynski J, Wieckowski MR, and Pinton P: Calcium signaling around Mitochondria Associated Membranes (MAMs). Cell Commun Signal CCS 9: 19, 2011.

28. Phillips MJ and Voeltz GK: Structure and function of ER membrane contact sites with other organelles. Nat Rev Mol Cell Biol 17: 69-82, 2015.

29. Ramming T and Appenzeller-Herzog C: Destroy and Exploit: Catalyzed Removal of Hydroperoxides from the Endoplasmic Reticulum. Int J Cell Biol 2013: e180906, 2013.

30. Ramming T, Hansen HG, Nagata K, Ellgaard L, and Appenzeller-Herzog C: GPx8 peroxidase prevents leakage of $\mathrm{H} 2 \mathrm{O} 2$ from the endoplasmic reticulum. Free Radic Biol Med 70: 106-116, 2014.

31. Ramming T, Okumura M, Kanemura S, Baday S, Birk J, Moes S, Spiess M, Jenö P, Bernèche S, Inaba K, and Appenzeller-Herzog C: A PDI-catalyzed thiol-disulfide 
switch regulates the production of hydrogen peroxide by human Ero1. Free Radic Biol Med.

32. Raturi A and Simmen T: Where the endoplasmic reticulum and the mitochondrion tie the knot: The mitochondria-associated membrane (MAM). Biochim Biophys Acta BBA - Mol Cell Res 1833: 213-224, 2013.

33. Raykhel I, Alanen H, Salo K, Jurvansuu J, Nguyen VD, Latva-Ranta M, and Ruddock L: A molecular specificity code for the three mammalian KDEL receptors. J Cell Biol 179: 1193-1204, 2007.

34. Rigoulet M, Yoboue ED, and Devin A: Mitochondrial ROS generation and its regulation: mechanisms involved in $\mathrm{H}(2) \mathrm{O}(2)$ signaling. Antioxid Redox Signal 14: 459468, 2011.

35. Rimessi A, Giorgi C, Pinton P, and Rizzuto R: The versatility of mitochondrial calcium signals: from stimulation of cell metabolism to induction of cell death. Biochim Biophys Acta 1777: 808-816, 2008.

36. Ronzoni R, Anelli T, Brunati M, Cortini M, Fagioli C, and Sitia R: Pathogenesis of ER Storage Disorders: Modulating Russell Body Biogenesis by Altering Proximal and Distal Quality Control. Traffic 11: 947-957, 2010.

37. Schäuble N, Lang S, Jung M, Cappel S, Schorr S, Ulucan Ö, Linxweiler J, Dudek J, Blum R, Helms V, Paton AW, Paton JC, Cavalié A, and Zimmermann R: BiP-mediated closing of the Sec61 channel limits Ca2+ leakage from the ER. EMBO J 31: 3282-3296, 2012.

38. Spiess M and Lodish HF: An internal signal sequence: The asialoglycoprotein receptor membrane anchor. Cell 44: 177-185, 1986.

39. Toppo S, Vanin S, Bosello V, and Tosatto SCE: Evolutionary and Structural Insights Into the Multifaceted Glutathione Peroxidase (Gpx) Superfamily. Antioxid Redox Signal 10: 1501-1514, 2008.

40. Utomo A, Jiang X, Furuta S, Yun J, Levin DS, Wang Y-CJ, Desai KV, Green JE, Chen P-L, and Lee W-H: Identification of a Novel Putative Non-selenocysteine Containing Phospholipid Hydroperoxide Glutathione Peroxidase (NPGPx) Essential for Alleviating Oxidative Stress Generated from Polyunsaturated Fatty Acids in Breast Cancer Cells. $J$ Biol Chem 279: 43522-43529, 2004. 
41. Van Coppenolle F, Vanden Abeele F, Slomianny C, Flourakis M, Hesketh J, Dewailly E, and Prevarskaya N: Ribosome-translocon complex mediates calcium leakage from endoplasmic reticulum stores. J Cell Sci 117: 4135-4142, 2004.

42. Vance JE: Phospholipid synthesis in a membrane fraction associated with mitochondria. J Biol Chem 265: 7248-7256, 1990.

43. Vance JE: MAM (mitochondria-associated membranes) in mammalian cells: Lipids and beyond. Biochim Biophys Acta BBA - Mol Cell Biol Lipids 1841: 595-609, 2014.

44. Vandecaetsbeek I, Vangheluwe P, Raeymaekers L, Wuytack F, and Vanoevelen J: The $\mathrm{Ca} 2+$ Pumps of the Endoplasmic Reticulum and Golgi Apparatus. Cold Spring Harb Perspect Biol 3: a004184, 2011.

45. Wang L, Zhang L, Niu Y, Sitia R, and Wang C: Glutathione Peroxidase 7 Utilizes Hydrogen Peroxide Generated by Ero1 $\alpha$ to Promote Oxidative Protein Folding. Antioxid Redox Signal 20: 545-556, 2013.

46. Wieckowski MR, Giorgi C, Lebiedzinska M, Duszynski J, and Pinton P: Isolation of mitochondria-associated membranes and mitochondria from animal tissues and cells. Nat Protoc 4: 1582-1590, 2009. 
Figure legends

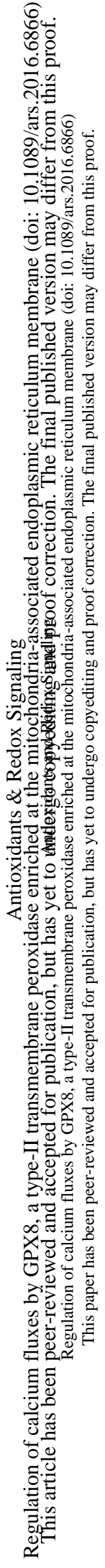




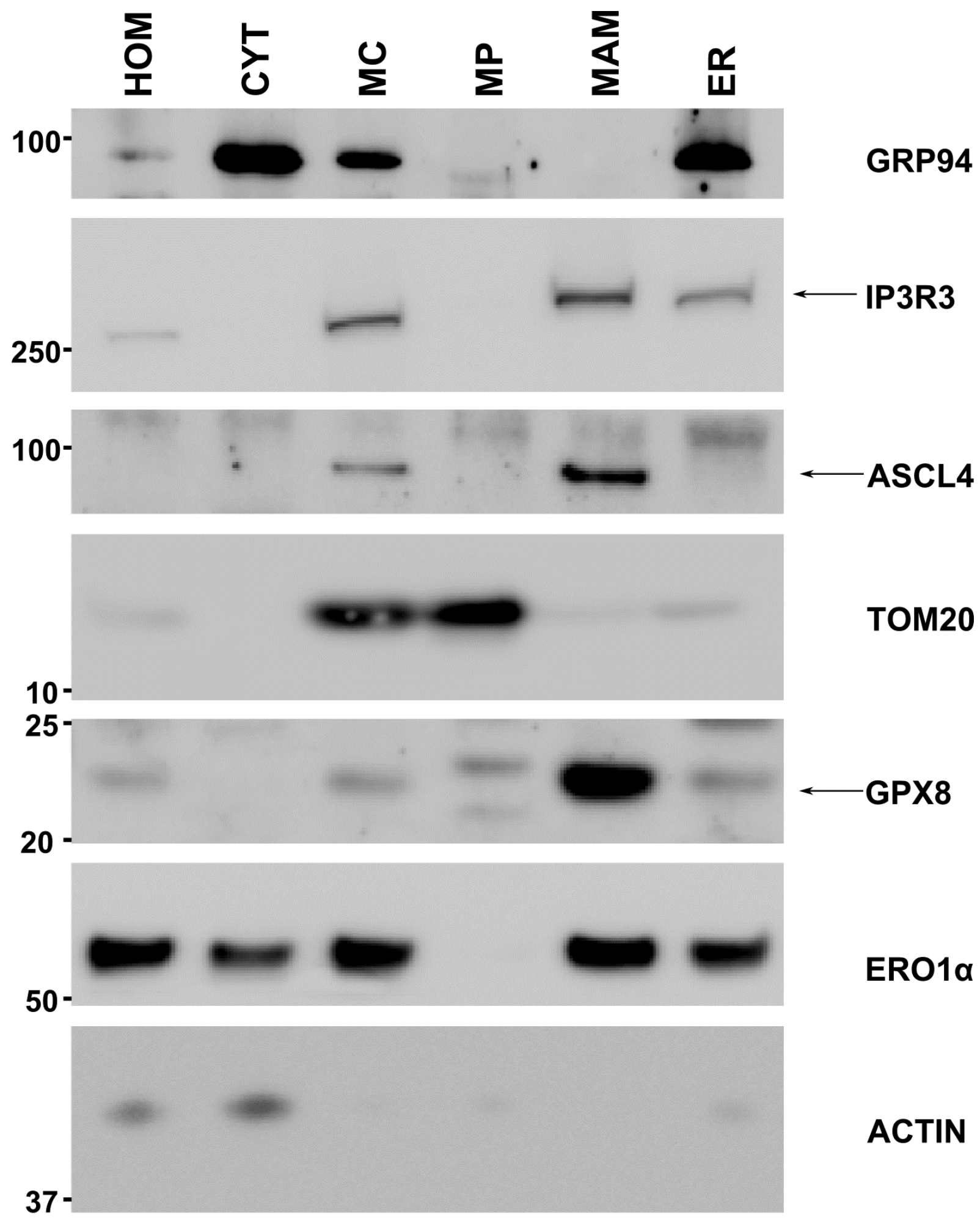

Figure 1: Endogenous GPX8 is highly enriched in MAM in HeLa cells.

HeLa cells homogenates (Hom) were fractionated by sequential centrifugation to obtain cytosol (Cyt), crude mitochondria (MC), pure mitochondria (MP), MAM and endoplasmic 
reticulum (ER) fractions. Equal amounts of proteins $(10 \mu \mathrm{g})$ from each fraction were resolved by reducing SDS-PAGE and analyzed by Western-blot using the indicated antibodies. Note the enrichment of GPX8 in MAM. Numbers on the left indicate the relative molecular weight based on co-migrating pre-stained molecular weight markers. (See supplementary figure S9 for uncropped western-blots). 
A

ER intraluminal $\mathrm{Ca}^{2+} \quad$\begin{tabular}{c} 
Histamine-induced \\
\cline { 2 - 3 }
\end{tabular} $\mathrm{ER} \mathrm{Ca}^{2+}$ extrusion rate
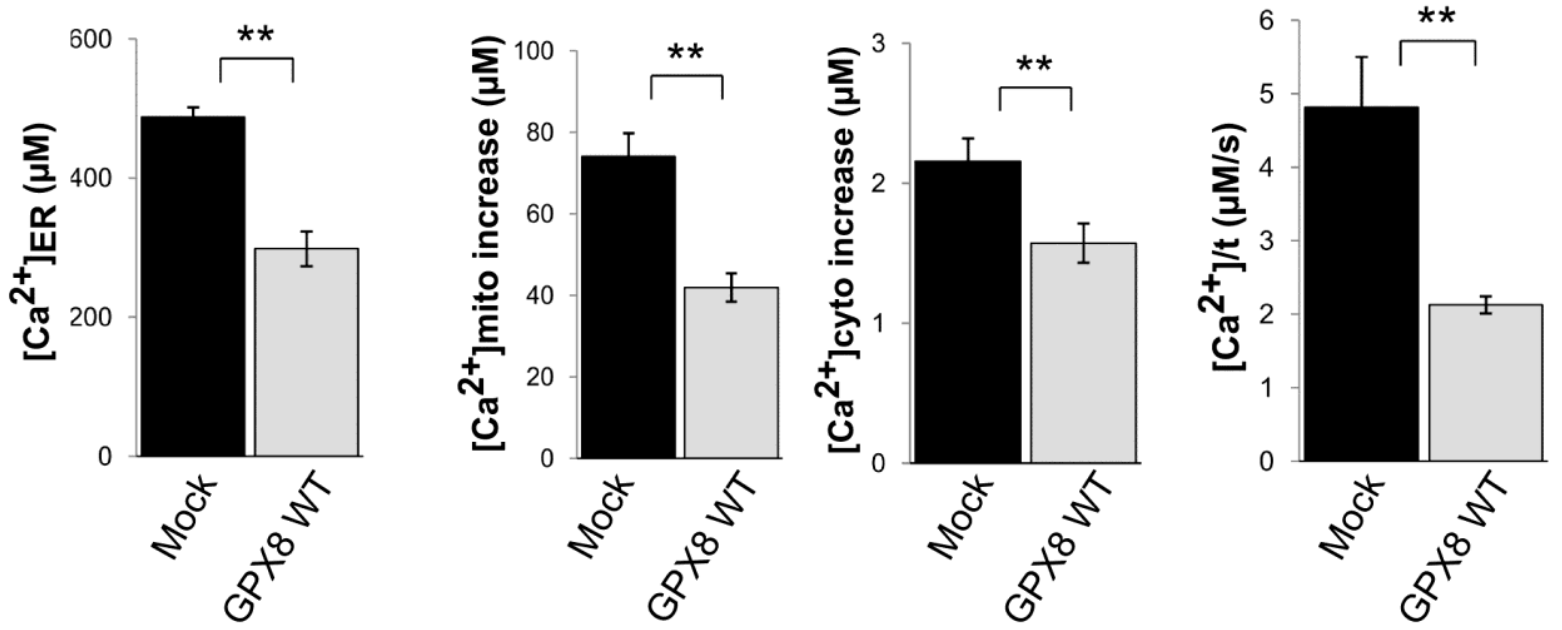

$\mathrm{B} /$
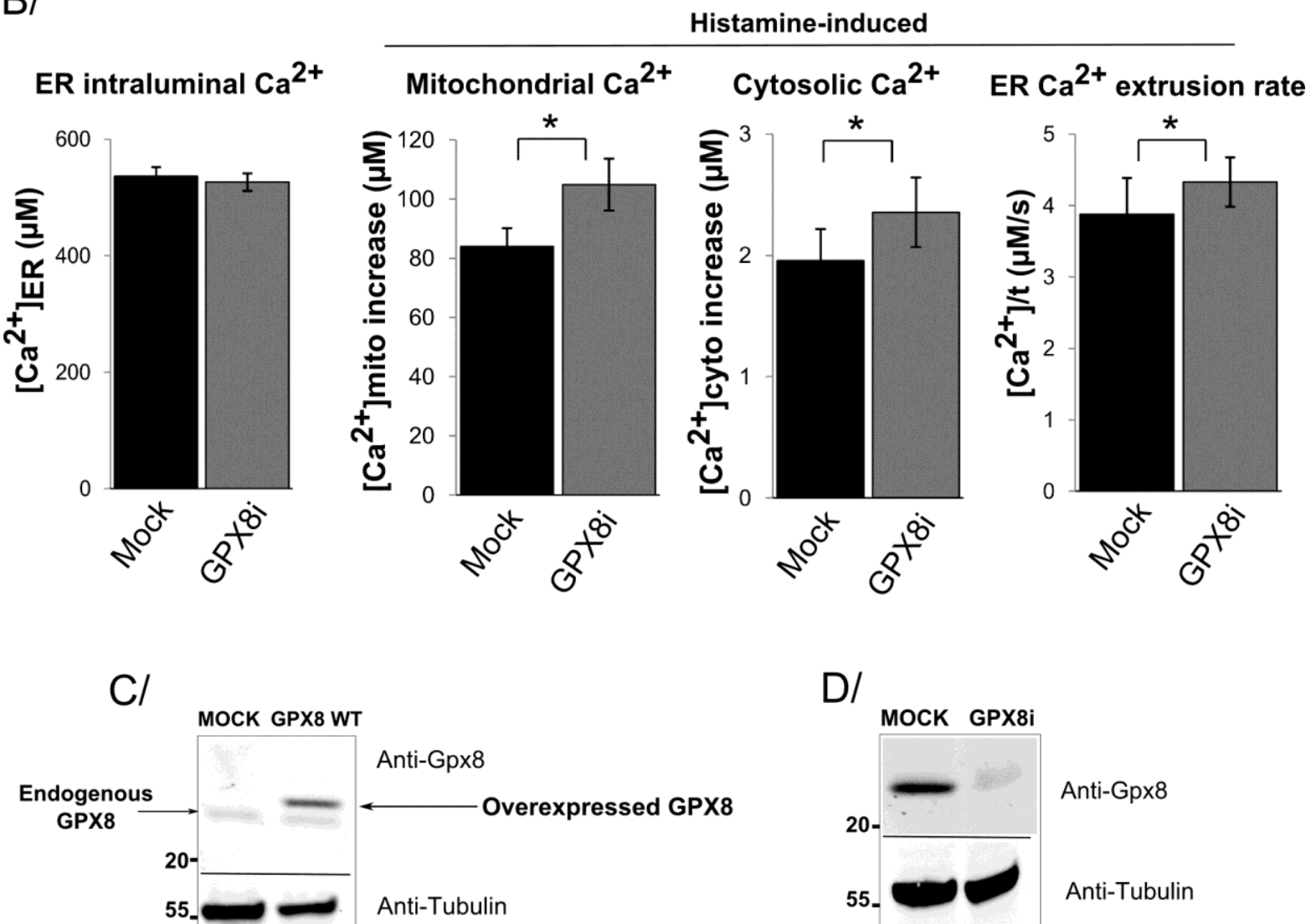

Figure 2: GPX8 level modulates ER $\mathrm{Ca}^{2+}$ concentration and fluxes.

(A) To monitor the effects of GPX overexpression on $\mathrm{Ca}^{2+}$ fluxes, HeLa cells were cotransfected with plasmids driving the expression of ER-, mitochondria- or cytosol-targeted 
aequorin probes and a HA tagged GPX8 (GPX8 WT) or an empty parental vector as a control (mock). After 36 hours of transfection, the indicated parameters were analyzed as described in materials and methods. The data are pooled from approximately 20 independent experiments and are expressed as the means $\pm \mathrm{SE},{ }^{*} \mathrm{p}<0.01$. (B) To down-regulate GPX8, HeLa cells expressing suitable aequorin probes (panel A) were transfected with a siRNA specific for GPX8 (GPX8i), or a negative control siRNA (mock) and analyzed after 36 hours. n=15 independent experiments, ${ }^{*} \mathrm{p}<0.05$. (C) and (D) are representative western-blot pictures showing GPX8 overexpression level and knockdown efficiency respectively. (See supplementary figure S10 for uncropped western-blots). 


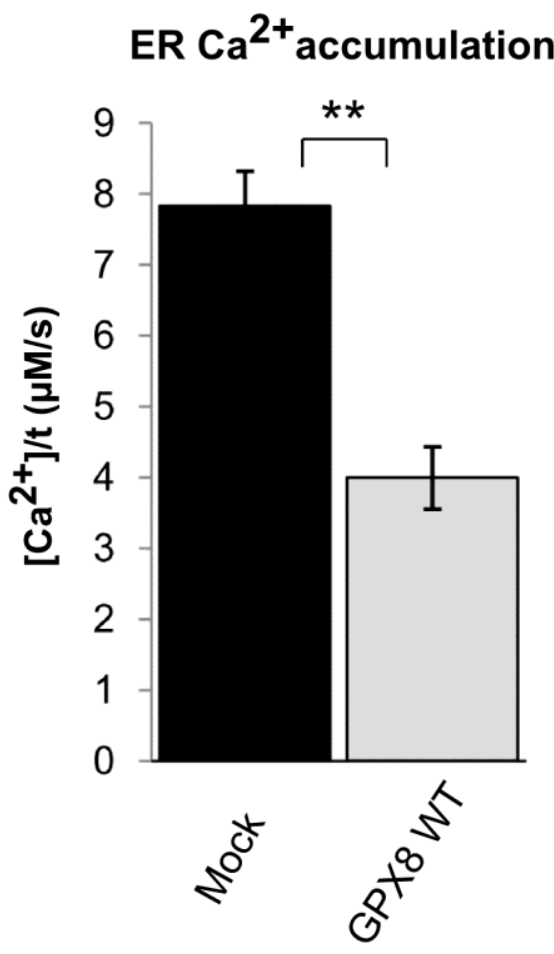

B/ ER Ca ${ }^{2+}$ accumulation rate

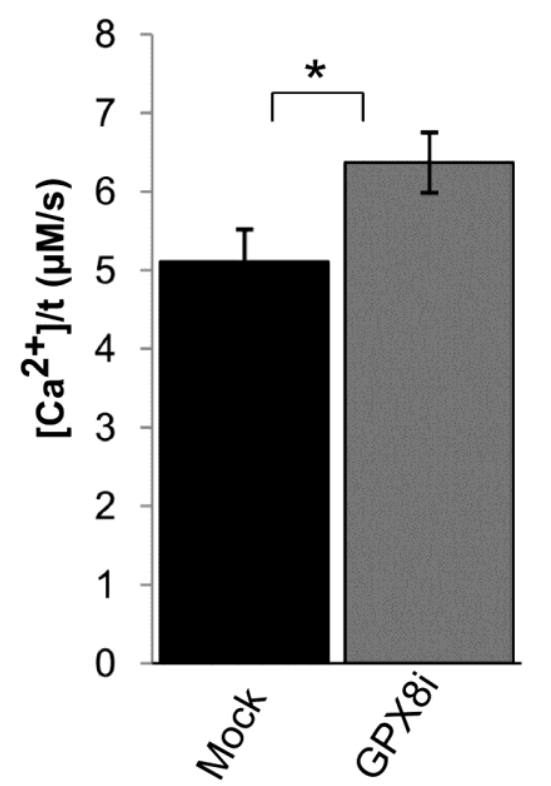

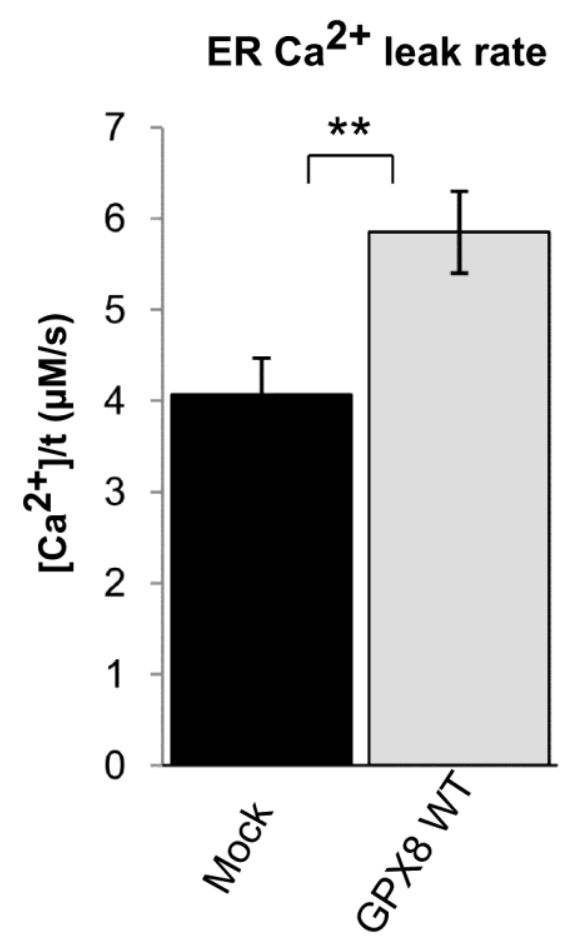

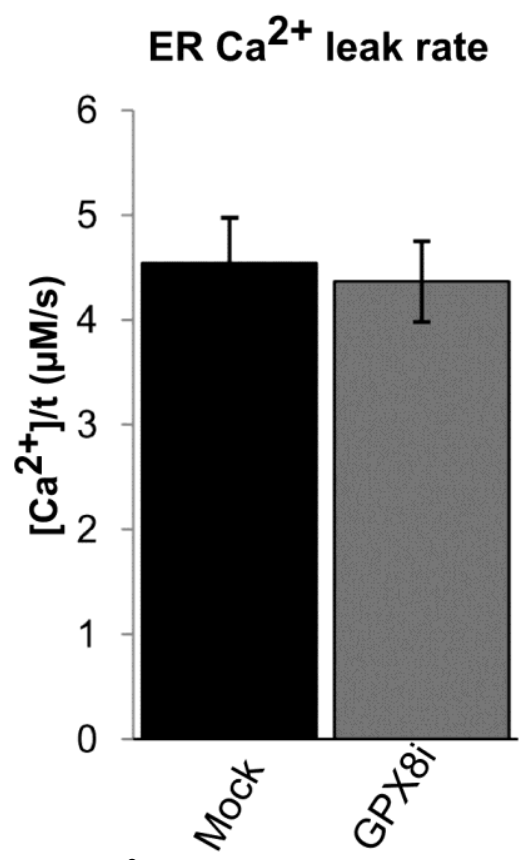

Figure 3: GPX8 level modulates the SERCA2b activity and ER $\mathrm{Ca}^{2+}$ leakage

HeLa cells overexpressing GPX8 (A) or treated with GPX8 siRNA (B) were analyzed as described in Figure 2 and the rates of $\mathrm{ER}^{\mathrm{Ca}^{2+}}$ refilling and the passive leak were measured as described in "materials and methods". The data are pooled from approximately 20 
independent experiments and are expressed as the means $\pm \mathrm{SE}, * * \mathrm{p}<0.01$ and $* \mathrm{p}<0.05$.

그를

जี

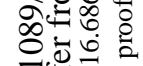

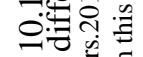

可高客

으응

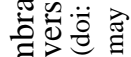

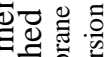

트를 믈

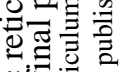

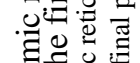

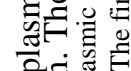

응형

ठ⿹𠃌

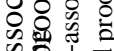

ont?

矛䮦: 
A

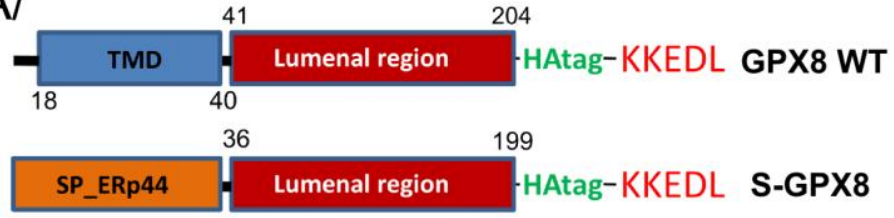

ृ्छ

으응

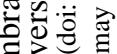

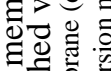

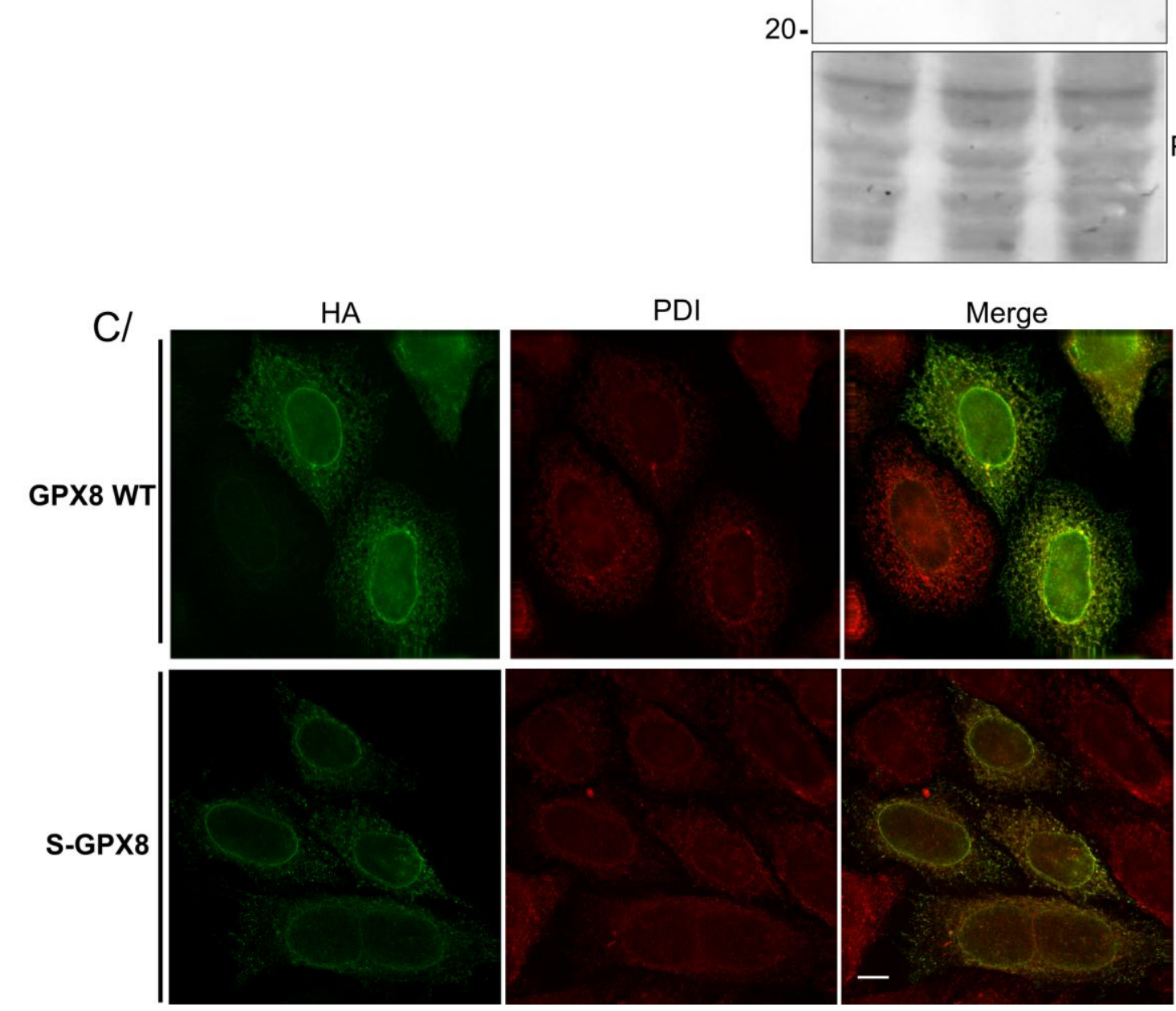

Figure 4: The GPX8-TMD is not necessary for its expression and ER localization

(A) Diagram summarizing the key features of the GPX8 variants utilized (GPX8-WT and SGPX8). The N-terminal 40 amino acids of GPX8, which include the transmembrane domain (TMD), were substituted by the cleavable leader peptide of the ER protein ERp44 (SP_ERp44).

B) Western-blot showing that the S-GPX8 variant is correctly expressed and cleaved, compared to the WT. Ponceau red staining assessed of protein loading. Numbers on the left indicate the relative molecular weight based on co-migrating pre-stained molecular weight 
markers. (See supplementary figure S11 for uncropped western-blots).

C) Immunofluorescence staining performed on fixed HeLa transfectants producing the GPX8 variants depicted in (A) co-stained with anti-HA (which recognizes the GPX8 transgene) and anti-PDI (Protein Disulfide Isomerase, used as ER marker) as antibodies. (Scale bar, $10 \mu \mathrm{m}$ )

(To see this illustration in color the reader is referred to the web version of this article at www.liebertonline.com/ars) 
A

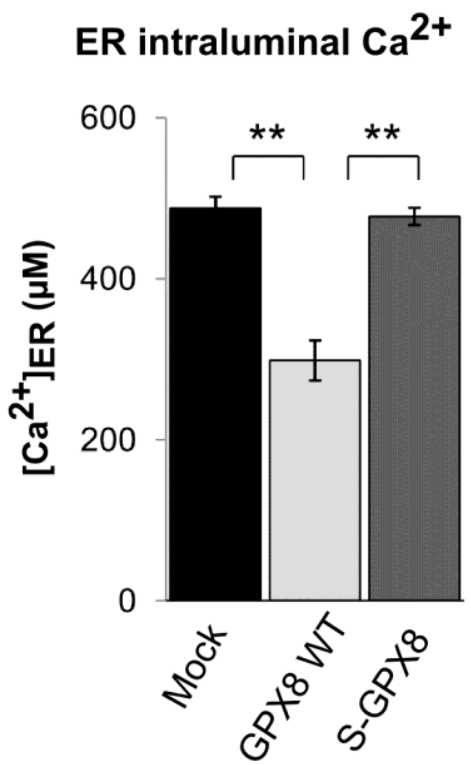

Histamine-induced

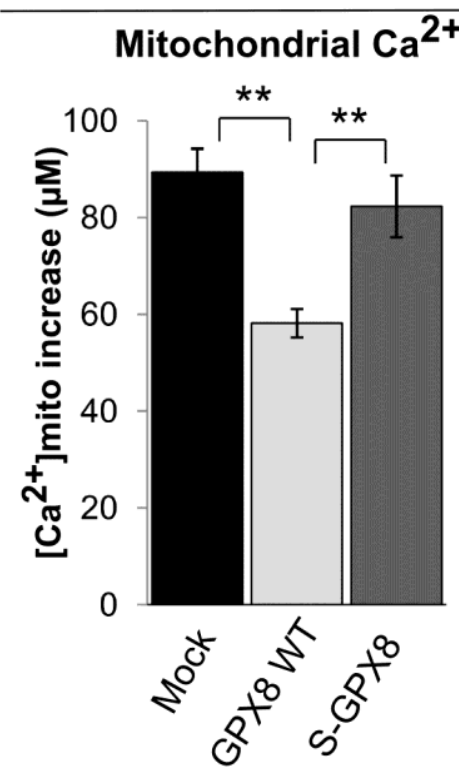

Cytosolic $\mathrm{Ca}^{2+}$

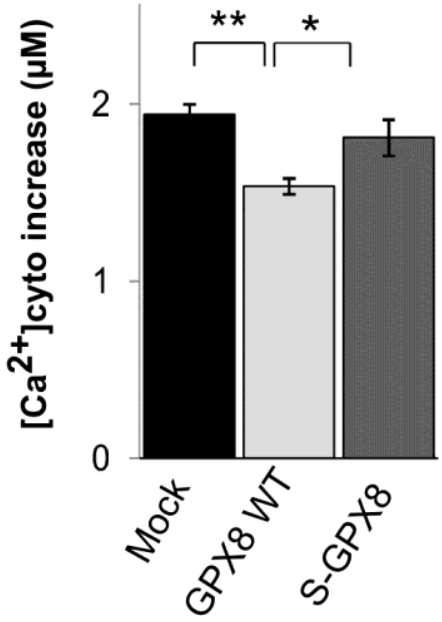

B/
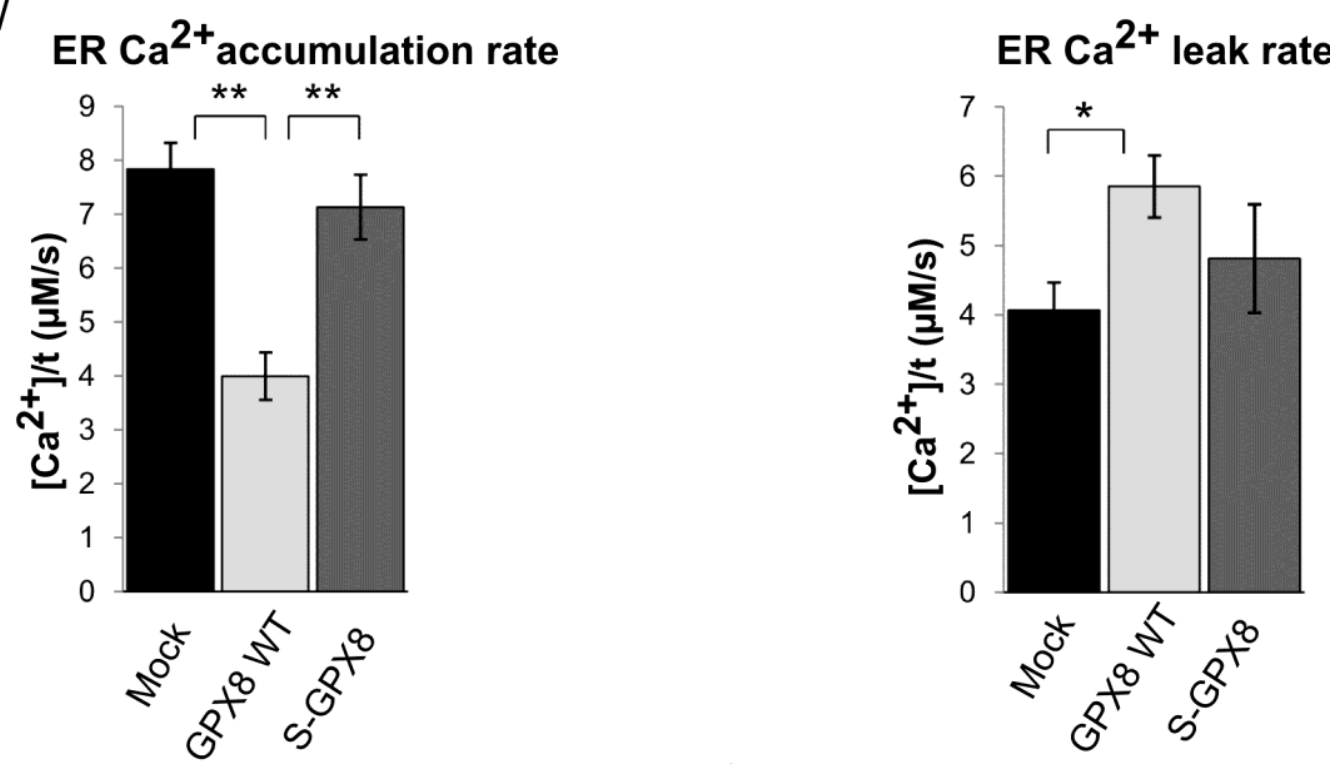

Figure 5: The GPX8 TMD is essential for $\mathrm{Ca}^{2+}$ dynamics regulation.

$\left[\mathrm{Ca}^{2+}\right]_{\mathrm{ER}}$, mitochondrial and cytosolic $\mathrm{Ca}^{2+}$ responses $(\mathrm{A})$ and rates of $\mathrm{ER}^{\mathrm{Ca}^{2+}}$ accumulation and passive leak (B) were determined in the indicated HeLa transfectants. The data are pooled from approximately 25 independent experiments and are expressed as the means $\pm \mathrm{SE}, * * \mathrm{p}<$ 0.01 and $* \mathrm{p}<0.05$ 
Page 36 of 46

ठ‥

0

굴

흐응

a.t.

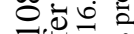

$0=0$

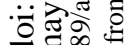

드을

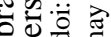

등

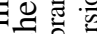

긍 를

司专

을

능 중

氜. ำ

음

응.응 웅

过司

ठㄴㅇㅇㅇㅠ

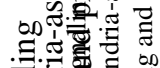

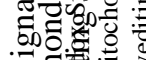

ن

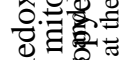

人ิ웡

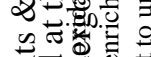

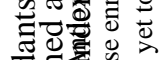

․ㅡㅁ음

可记递

$<$ 讨

플 땡

응흘 을

口두

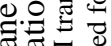

궁

중을

菏

춘

$=0$

10

خ㓉

踣

人류

(궁

구웛유.

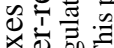

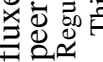

륭

ชี่

응

으른

츨.

of 

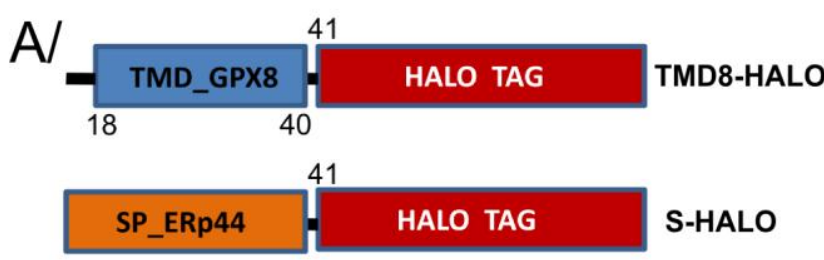

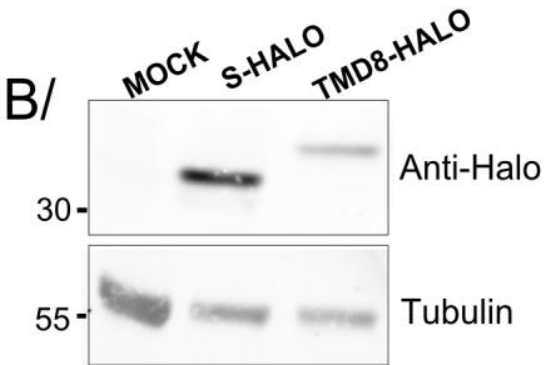

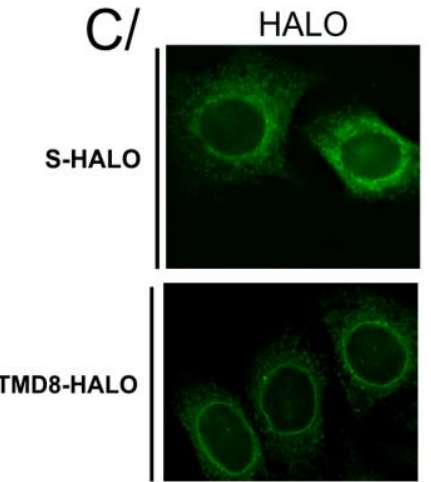

$\mathrm{D} /$

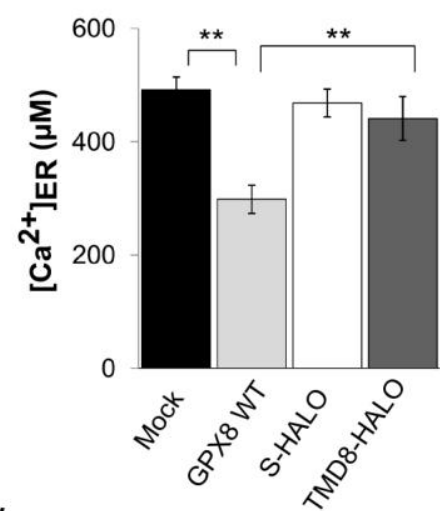

E/

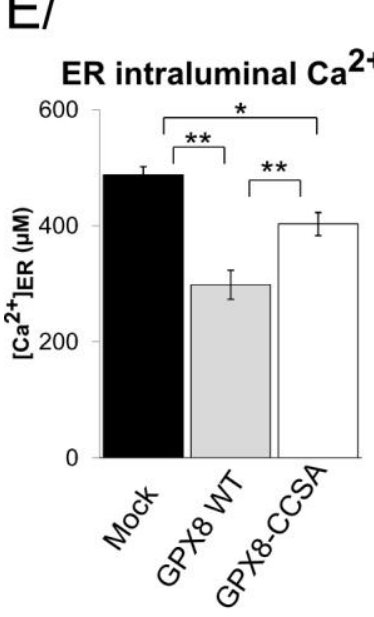

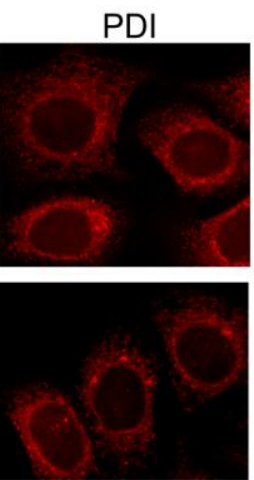

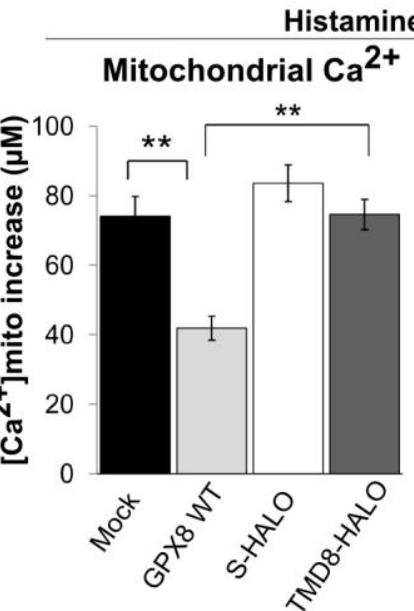

Histamine-induced

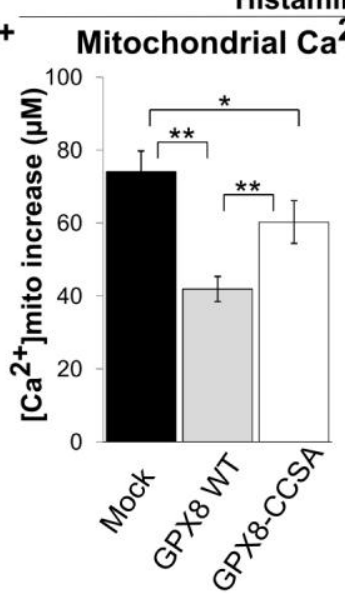

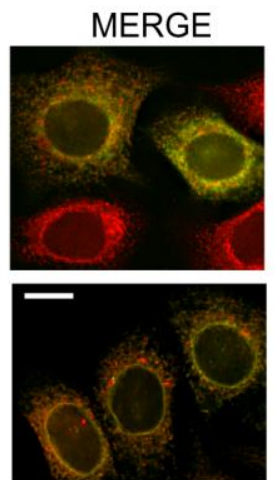

Histamine-induced

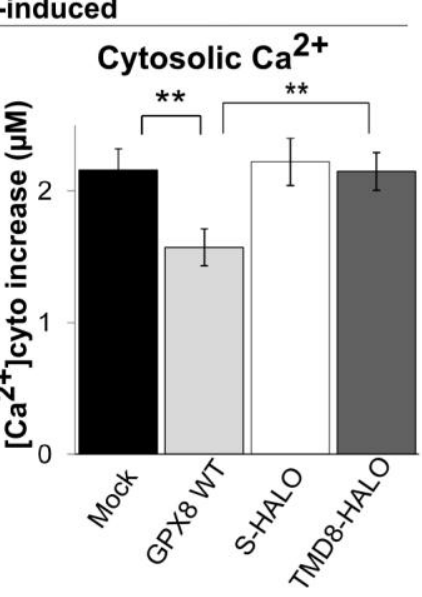

Figure 6: GPX8 peroxidatic cysteines are involved in $\mathrm{Ca}^{2+}$ regulation.

(A) Diagram illustrating the constructs used in the following assays (B-D). As previously 
described (22), S-HALO drives the targeting of the Halotag to the ER via the ERp44 cleavable ER signal peptide (SP_ERp44). In TMD8-HALO, the Halotag had been extended with the ER targeting region (1-40) of GPX8, which includes its transmembrane domain (TMD_GPX8). Both proteins contain C-terminal RDEL motifs (not depicted here) for mediating their ER localization.

(B) Western-blot showing S-HALO and C8-TMD8-HALO protein expression levels. Protein extracts of the corresponding HeLa transfectants were separated by SDS-PAGE and analyzed by Western-blotting with the indicated antibodies. (See supplementary figure S12 for uncropped western-blots).

C) Fluorescence microscopy images of fixed transient HeLa transfectants expressing SHALO and TMD8-HALO. Halo signals were obtained by incubating cells with R110, a fluorescent specific ligand of the HALO protein, while PDI was visualized with an anti-PDI antibody. (Scale bar, $10 \mu \mathrm{m})$

D) HeLa cells were co-transfected with plasmids encoding S-HALO or TMD8-HALO or the empty parental vector. The $\left[\mathrm{Ca}^{2+}\right]_{\mathrm{ER}}$, mitochondrial and cytosolic $\mathrm{Ca}^{2+}$ responses were monitored as described in the "material and methods" section 36 hours after transfection. The data are pooled from approximately 15 independent experiments and are expressed as the means $\pm \mathrm{SE}, * * \mathrm{p}<0.01$.

E) Western-blot illustrating the expression of GPX8 WT and GPX8-CCSA (GPX8 with Cys79 and 108 mutated in Ser and Ala respectively). Numbers on the left indicate the relative molecular weight based on co-migrating pre-stained molecular weight markers. (See supplementary figure S12 for uncropped western-blots).

F) HeLa cells were co-transfected with plasmids encoding GPX8-CCSA, GPX8-WT or empty parental vector, as well as organelle-specific aequorin probes. $\left[\mathrm{Ca}^{2+}\right]_{\mathrm{ER}}$, mitochondrial and 
cytosolic $\mathrm{Ca}^{2+}$ responses were monitored as described in" materials and method" section, $\mathrm{n}=25$ independent experiments, $* * \mathrm{p}<0.01$ and $* \mathrm{p}<0.05$.

(To see this illustration in color the reader is referred to the web version of this article at www.liebertonline.com/ars) 

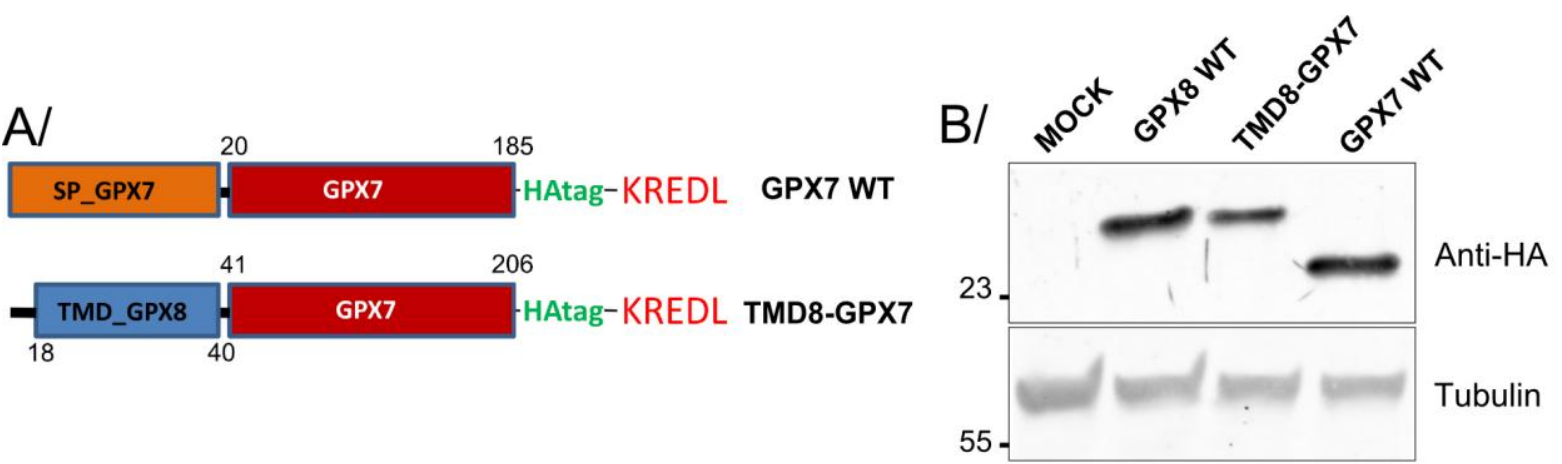
C/

HA

PDI

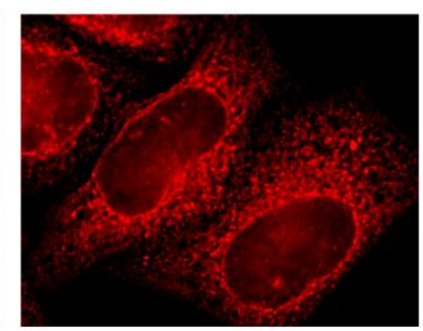

MERGE
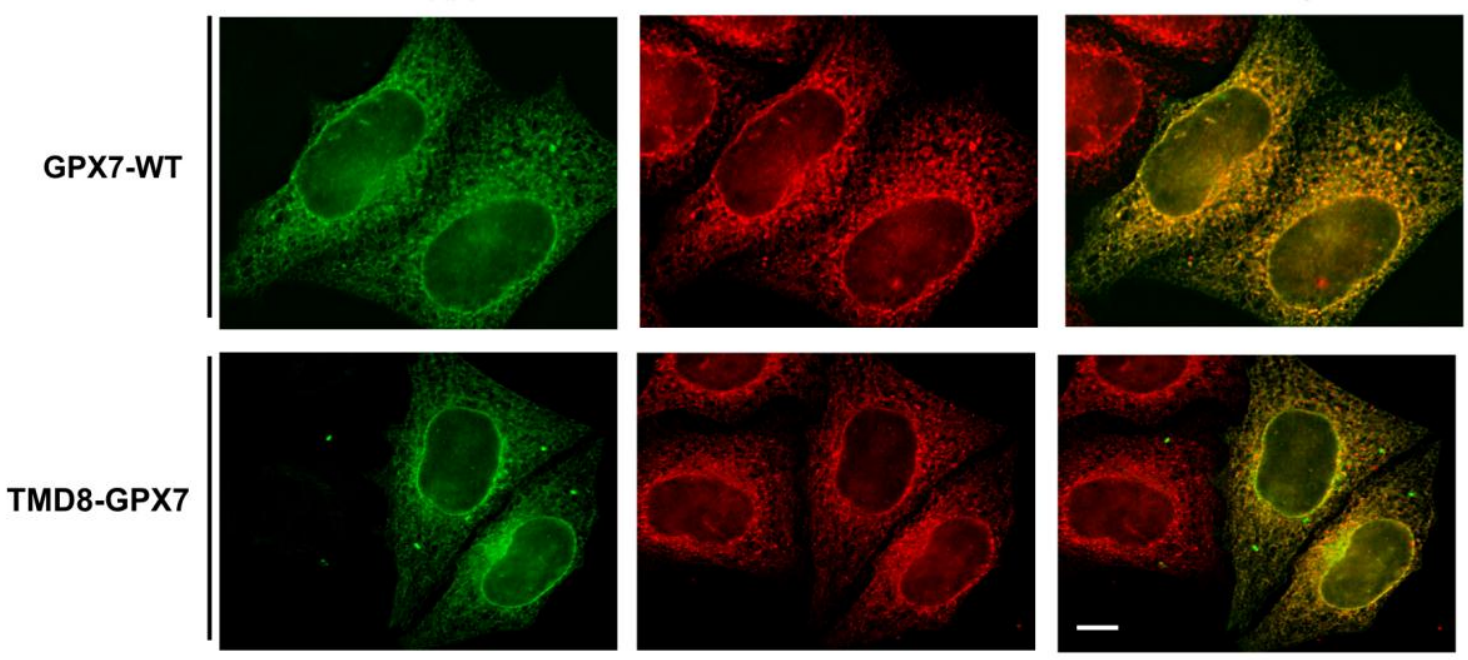

D/
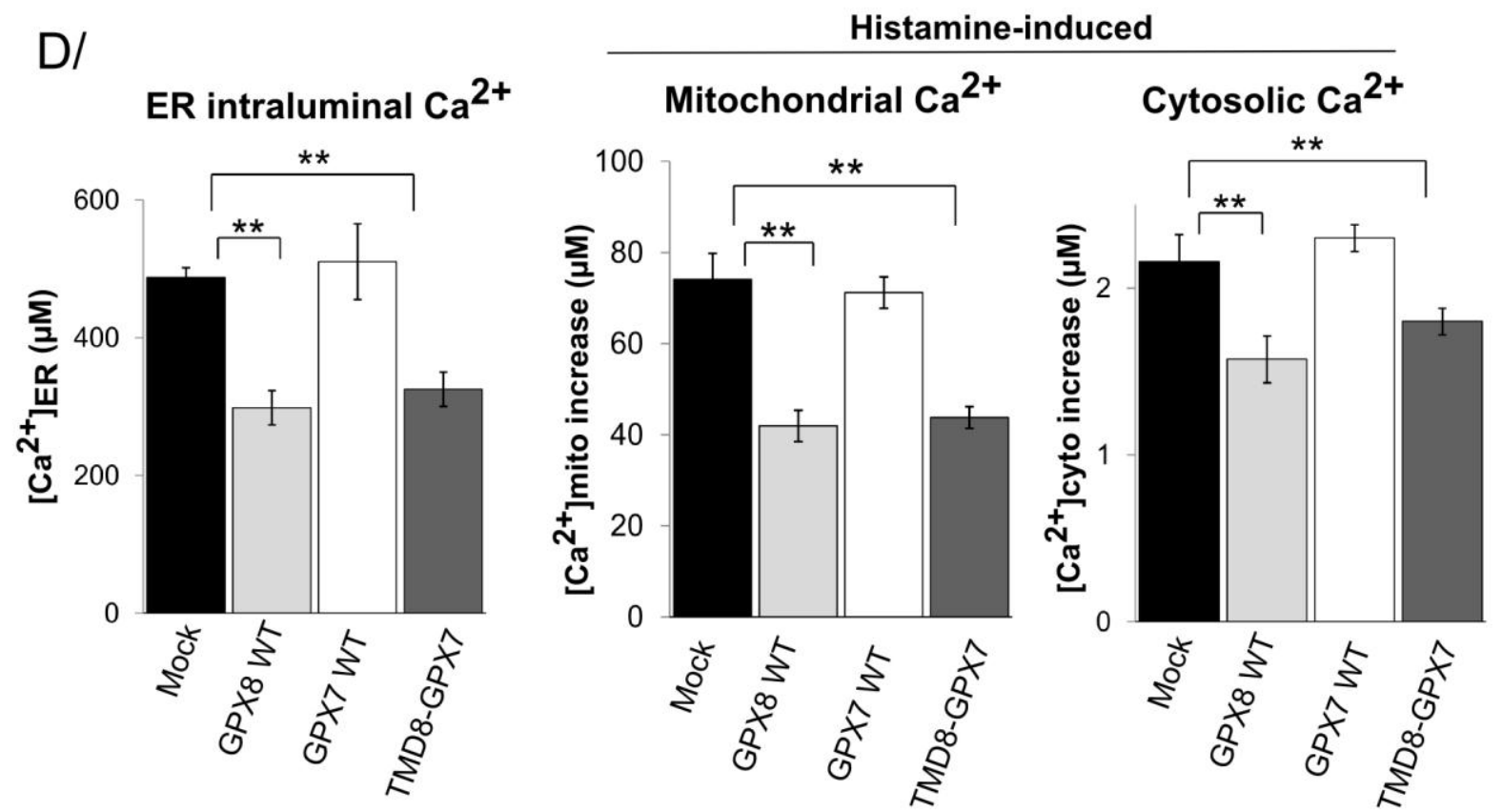

Figure 7: GPX7 regulates $\mathrm{Ca}^{2+}$ dynamics only if fused to the GPX8 transmembrane 


\section{domain}

(A) Diagram illustrating the main differences between GPX7-WT and the TMD8-GPX7 variants. In TMD8-GPX7, the regular and cleavable signal peptide of GPX7 (SP_GPX7) has been substituted by the GPX8 ER targeting region (1-40), which includes the TMD (TMD_GPX8).

(B) Western-blot analyses confirming the expression of GPX8-WT, GPX7-WT and TMD8GPX7. Numbers on the left indicate the relative molecular weight based on co-migrating prestained molecular weight markers. (See supplementary figure S13 for uncropped westernblots).

(C) Immunofluorescence staining performed on fixed HeLa cells transiently expressing GPX7-WT or TMD8-GPX7 as indicated. Cells have been double-stained with anti-HA and anti-PDI antibodies. (Scale bar, $10 \mu \mathrm{m}$ )

(D) HeLa cells were co-transfected with plasmids encoding GPX8-WT, GPX7-WT, TMD8GPX7 or the corresponding empty parental vector, as well as organelle specific aequorin probes. $\left[\mathrm{Ca}^{2+}\right]_{\mathrm{ER}}$, mitochondrial and cytosolic $\mathrm{Ca}^{2+}$ responses were monitored as described in "materials and method" section. The data are pooled from approximately 15 independent experiments and are expressed as the means $\pm \mathrm{SE}, * * \mathrm{p}<0.01$.

(To see this illustration in color the reader is referred to the web version of this article at www.liebertonline.com/ars) 

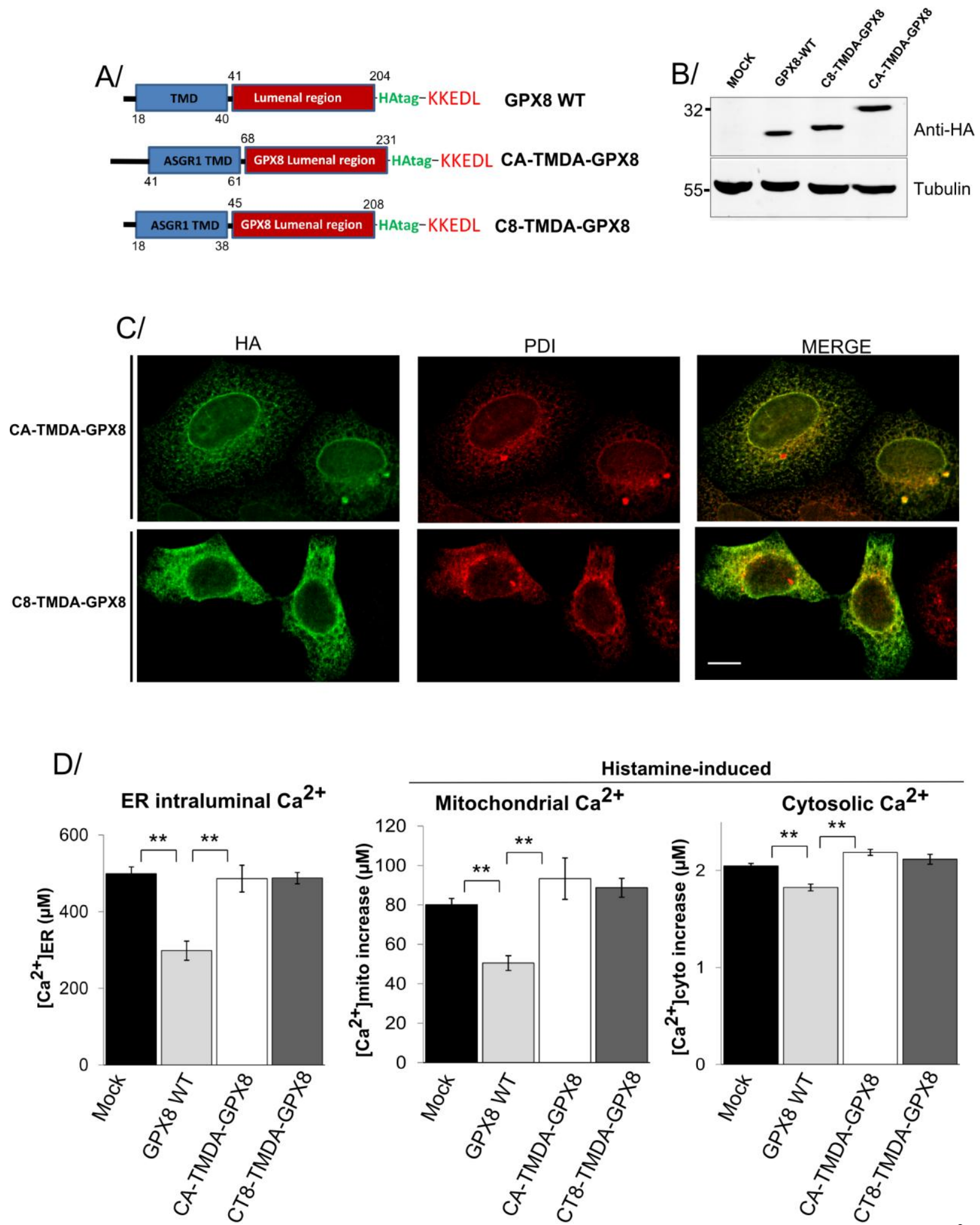

Figure 8: GPX8 N terminus hydrophobic stretch is specifically required for GPX8 $\mathrm{Ca}^{2+}$ regulatory properties.

(A) Like in the other figures, the diagram summarizes the main features of the GPX8 variants 
used. In CA-TMDA-GPX8, the first 40 amino acids of GPX8, which include its TMD, was replaced by the N-terminal region (residues 1-61) of the ASGR1 receptor, which is also a type II transmembrane protein with its TMD (TMD_ASGR1) spanning from residues 41 to 61 . In C8-TMDA-GPX8, the cytosolic N-terminal tip of GPX8 has been conserved while only the TMD of GPX8 has been substituted with the one of ASGR1. The Western-blot in (B) shows expression levels of GPX8-WT, CA-TMDA-GPX8 and C8-TMDA-GPX8. (See supplementary figure S14 for uncropped western-blots).

C) Fixed HeLa cells transiently expressing CA-TMDA-GPX8 (upper panels) and C8-TMDAGPX8 (lower panels) were double-stained with anti-HA and anti-PDI antibodies. (Scale bar, $10 \mu \mathrm{m})$

D) HeLa cells were co-transfected with plasmids encoding GPX8-WT, CA-TMDA-GPX8, C8-TMDA-GPX8 and the corresponding empty parental vector, as well as organelle specific aequorin probes. $\left[\mathrm{Ca}^{2+}\right]_{\mathrm{ER}}$, mitochondrial and cytosolic $\mathrm{Ca}^{2+}$ responses were monitored as described in materials and method section. The data are pooled from approximately 15 independent experiments and are expressed as the means $\pm \mathrm{SE}, * * \mathrm{p}<0.01$.

(To see this illustration in color the reader is referred to the web version of this article at www.liebertonline.com/ars) 


\section{Supplementary figures legends}

\section{Figure S1 (related to figure 2): GPX8 level modulates ER $\mathrm{Ca}^{2+}$ concentration and fluxes}

Representative traces for mitochondrial (left panels), cytosolic (central panels) and ER Ca concentrations measures in cells overexpressing (A) or silenced for (B) GPX8. Where indicated, HeLa cells were challenged with $100 \mu \mathrm{M}$ histamine to induce IP3-dependent $\mathrm{Ca}^{2+}$ transients. In ER measurements, 36 hours after transfection the $\mathrm{Ca}^{2+}$ stores were depleted of $\mathrm{Ca}^{2+}$ to optimize aequorin reconstitution as detailed in the materials and methods section. After reconstitution, the cells were transferred to the luminometer chamber, and the ER store was refilled by switching the perfusion medium from KRB/EGTA to KRB containing $1 \mathrm{mM}$ $\mathrm{CaCl}_{2}$.

\section{Figure S2 (related to figure 2): GPX8 overexpression decreases ER $\mathrm{Ca}^{2+}$ dynamics}

ER calcium was measured in HeLa cells overexpressing (GPX8 WT) or not (mock) GPX8 using the FRET-based probe D1ER (see "supplementary experimental procedures" section for details). Right panel is a representative trace of the measurements.

Figure S3 (related to figure 2): GPX8 impairs $\mathrm{H}_{2} \mathrm{O}_{2}$-dependant intracellular $\mathrm{Ca}^{2+}$ mobilization.

HeLa cells overexpressing (GPX8 WT) or not (mock) GPX8 were treated with $\mathrm{H}_{2} \mathrm{O}_{2}(1 \mathrm{mM})$ and intracellular $\mathrm{Ca}^{2+}$ was measured using the Fura-2/AM probe (see "supplementary experimental procedures" section for details). The right panel shows a representative trace of the measurements.

Figure S4: GPX8 TMD-dependent modulation of $\mathrm{Ca}^{2+}$ dynamics in (A) SH-SY5 (neuroblastoma) and (B) A549 (lung) cells.

SH-SY5 and A549 cells were either co-transfected with ER, mitochondrial and cytosolic 
specific targeted-probes and the different constructs indicated. The bars show the extent of the increase of the ER, mitochondrial and cytosolic $\left[\mathrm{Ca}^{2+}\right]$ peak induced by $\mathrm{Ca}^{2+} 1 \mathrm{mM}$ refilling (ER intraluminal content) and by bradykinin (SH-SY5) or histamine (A549) agonist stimulation.

\section{Figure S5: The GPX8 transmembrane is not necessary for its MAM enrichment}

(A) Diagram summarizing the key features of the GPX8 variants utilized (see figure 4 legend and text for details) (B) Homogenates (Hom) of HeLa cells stably expressing HA-tagged GPX8-WT or the S-GPX8 variant were fractionated by differential centrifugation to obtain cytosol (Cyt), pure mitochondria (MP), MAM and endoplasmic reticulum (ER) fractions. Equal protein quantities from each fraction were resolved by reducing SDS-PAGE and analyzed by western-blot using the indicated antibodies. For the "anti-HA" panels, two different exposures (for better visualizing the HA signal of the S-GPX8 protein) of the same membrane have been assembled here (as indicated by the separating black line). (See supplementary figure S15 for uncropped western-blots).

Figure S6: The basal mitochondrial $\mathrm{Ca}^{2+}$ level is not influenced by the expression of GPX8 and its different variants

Basal mitochondrial $\mathrm{Ca}^{2+}$ concentrations were assessed by imaging 2mt-GCaMP6mexpressing cells, as described in experimental procedures. HeLa cells were either cotransfected with 2mt-GCaMP6m and GPX8 WT or the indicated constructs (upper panel). The values are expressed as ratios between the excitation wavelengths of 474 and $410 \mathrm{~nm}$, which are proportional to the $\mathrm{Ca}^{2+}$ concentration and independent of probe expression levels. The lower panel depicts the result of the same experiment performed in HeLa cells silenced for GPX8.

Figure S7: GPX8 overexpression does not decrease the level of $\mathrm{Ca}^{2+}$ related transporters 
The expression of IP3R1, IP3R3, MCU, SERCA2 and HA-tagged GPX8-WT was analyzed by Western-blot with specific antibodies on protein extracts of HeLa cells overexpressing GPX8 (GPX8 WT) or not (mock). Ponceau red staining was used to assess protein loading. (See supplementary figure S16 for uncropped western-blots).

\section{Figure S8: Interaction between GPX8 and SERCA2}

Lysates of cells expressing Flag-tagged variants of GPX8 WT or S-GPX8 or a parental empty vector (mock) were immunoprecipitated with anti-FLAG M2 agarose gel (Sigma Aldrich) and analyzed by Western blot with antibodies against FLAG tag and SERCA2. 5\% of the lysate before immunoprecipation was similarly analyzed in parallel (input). (See supplementary figure S17 for uncropped western-blots). 

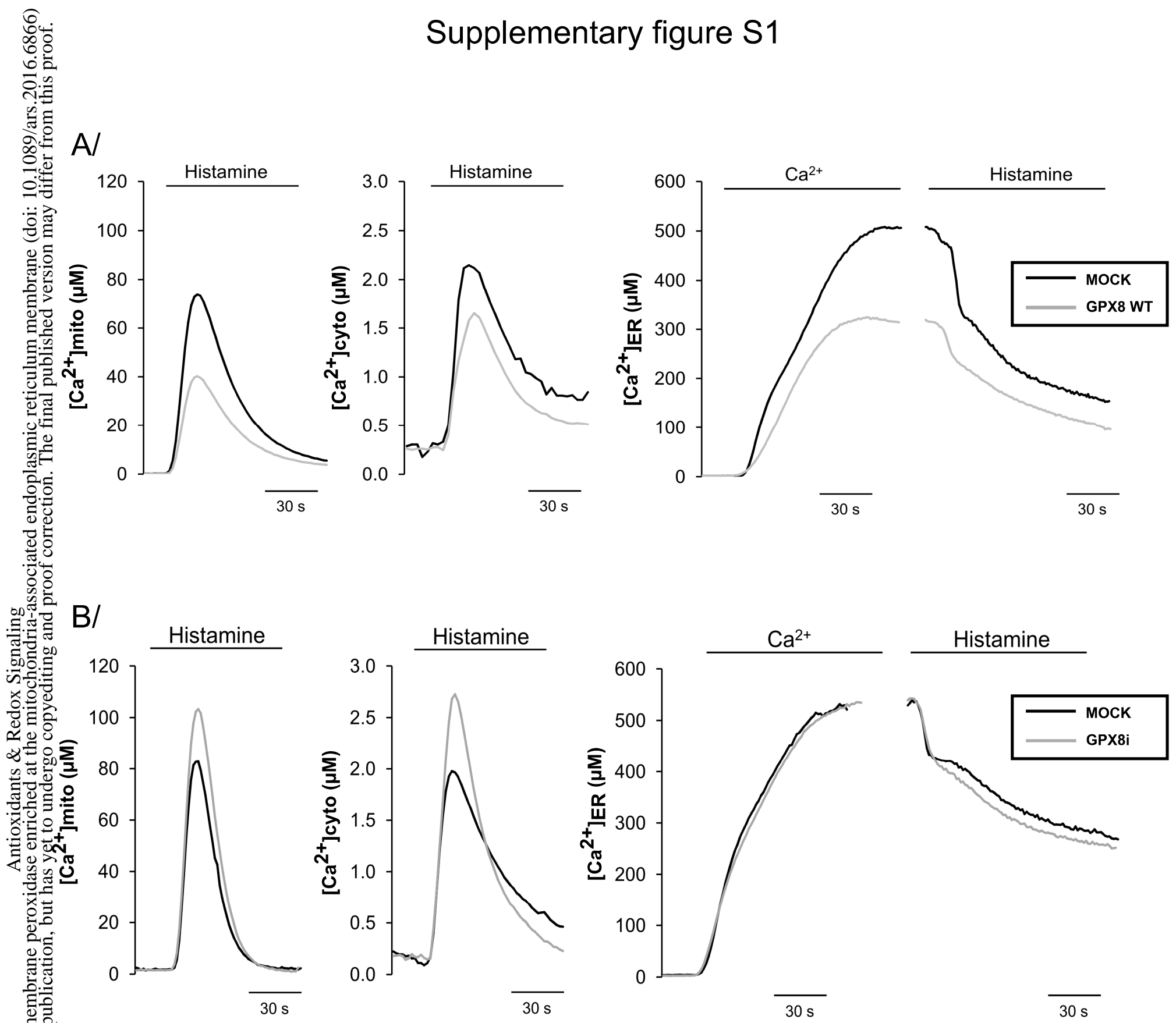


\section{Supplementary figure S2}
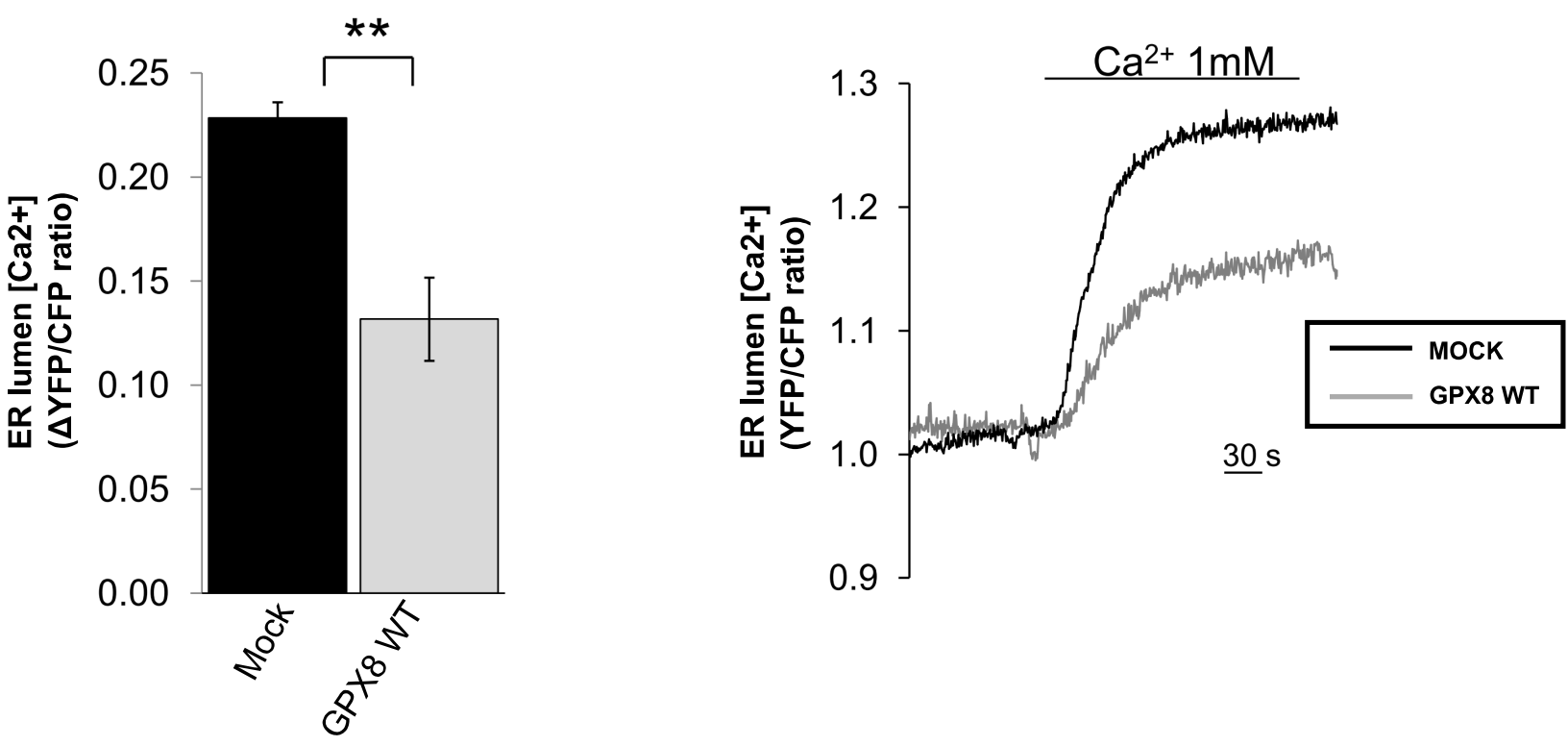
Supplementary figure S3
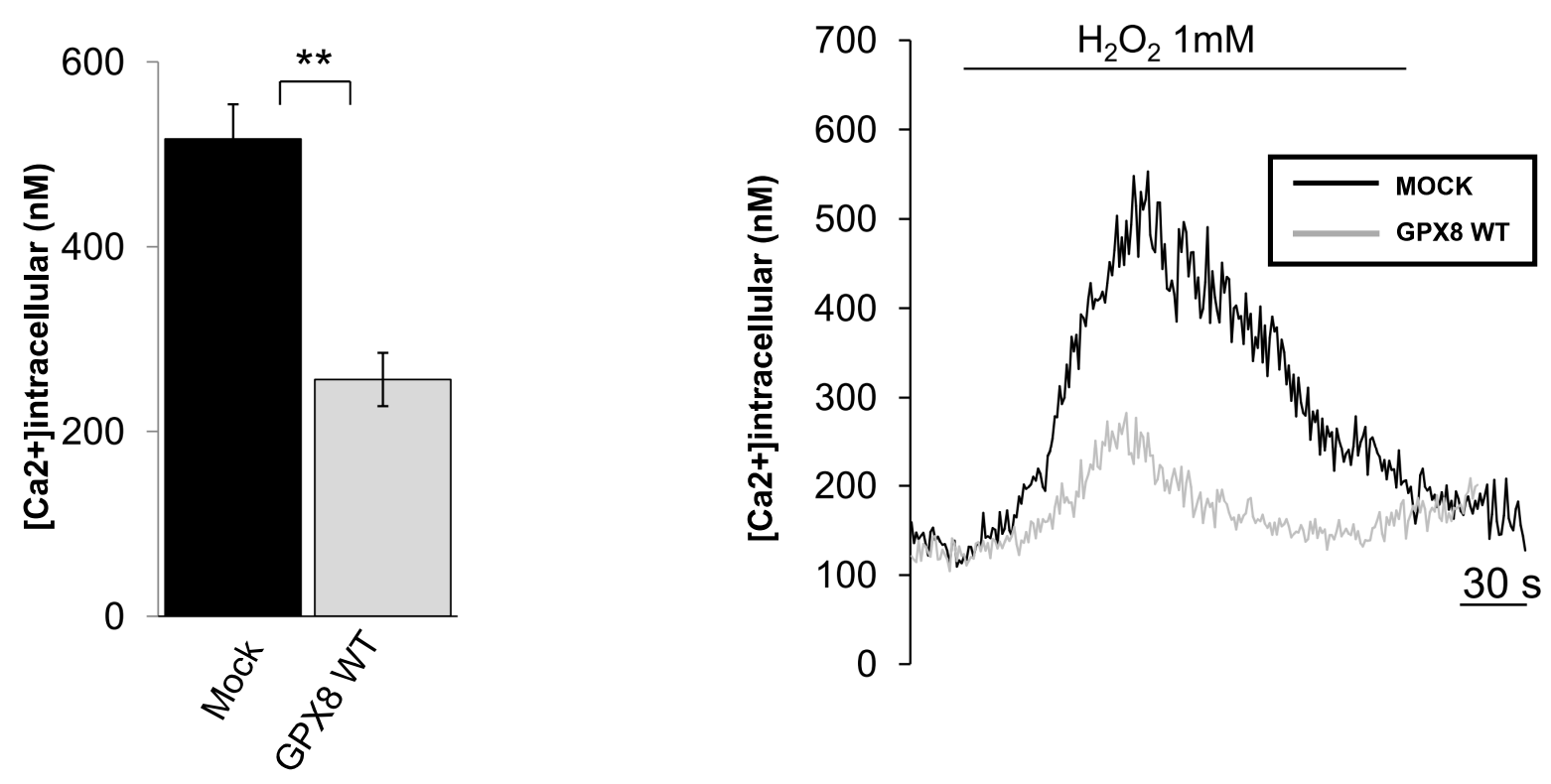


\section{Supplementary figure S4}

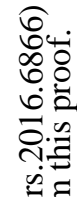

\section{สี้}

$\stackrel{ \pm}{0}$

迅
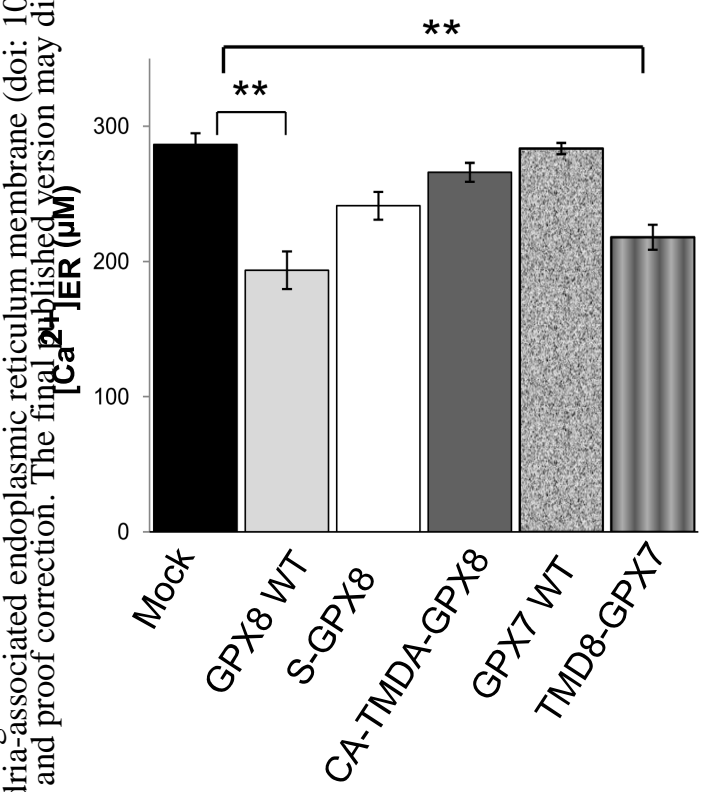

ER intraluminal $\mathrm{Ca}^{2+}$
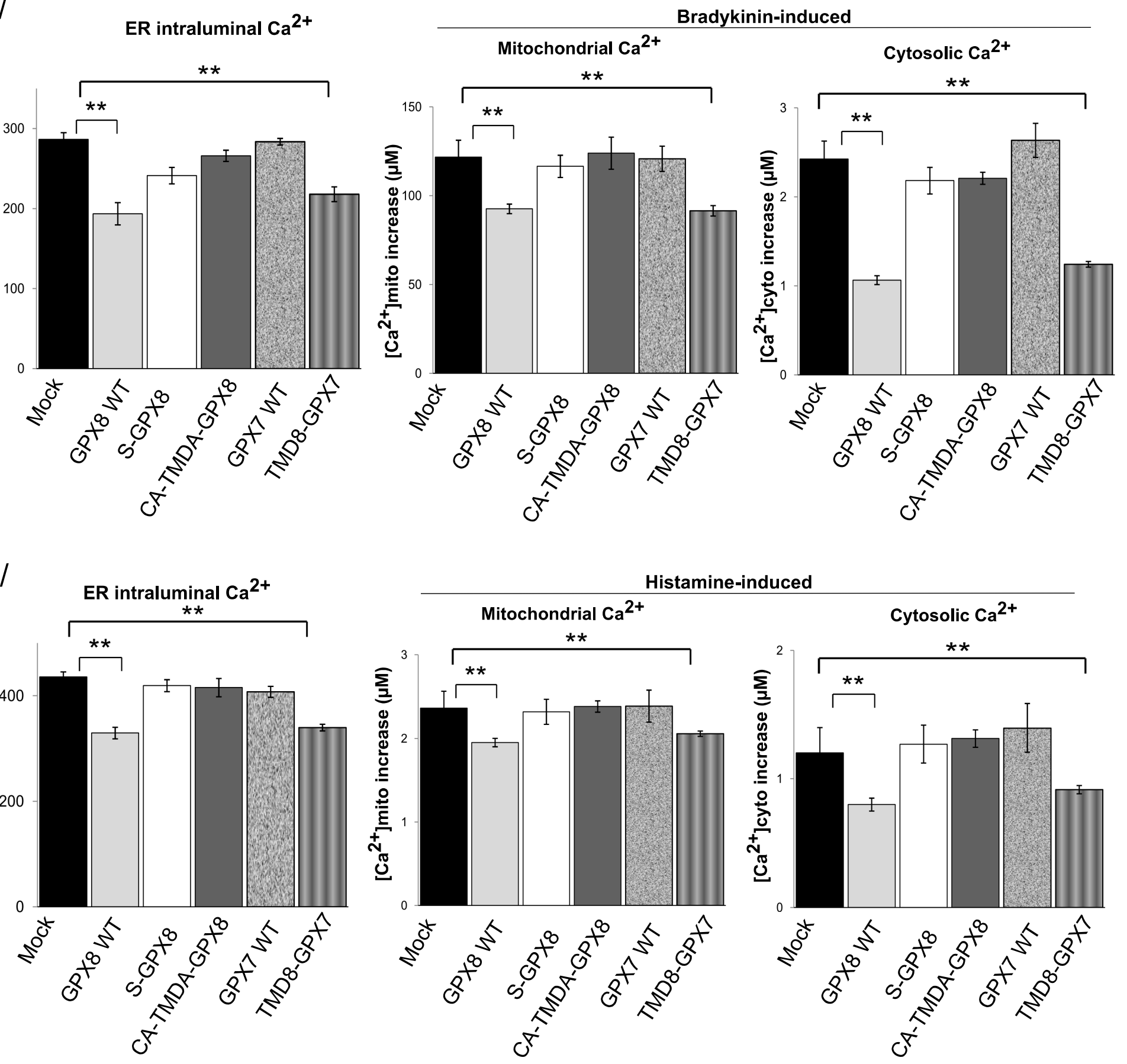


\section{Supplementary figure S5}

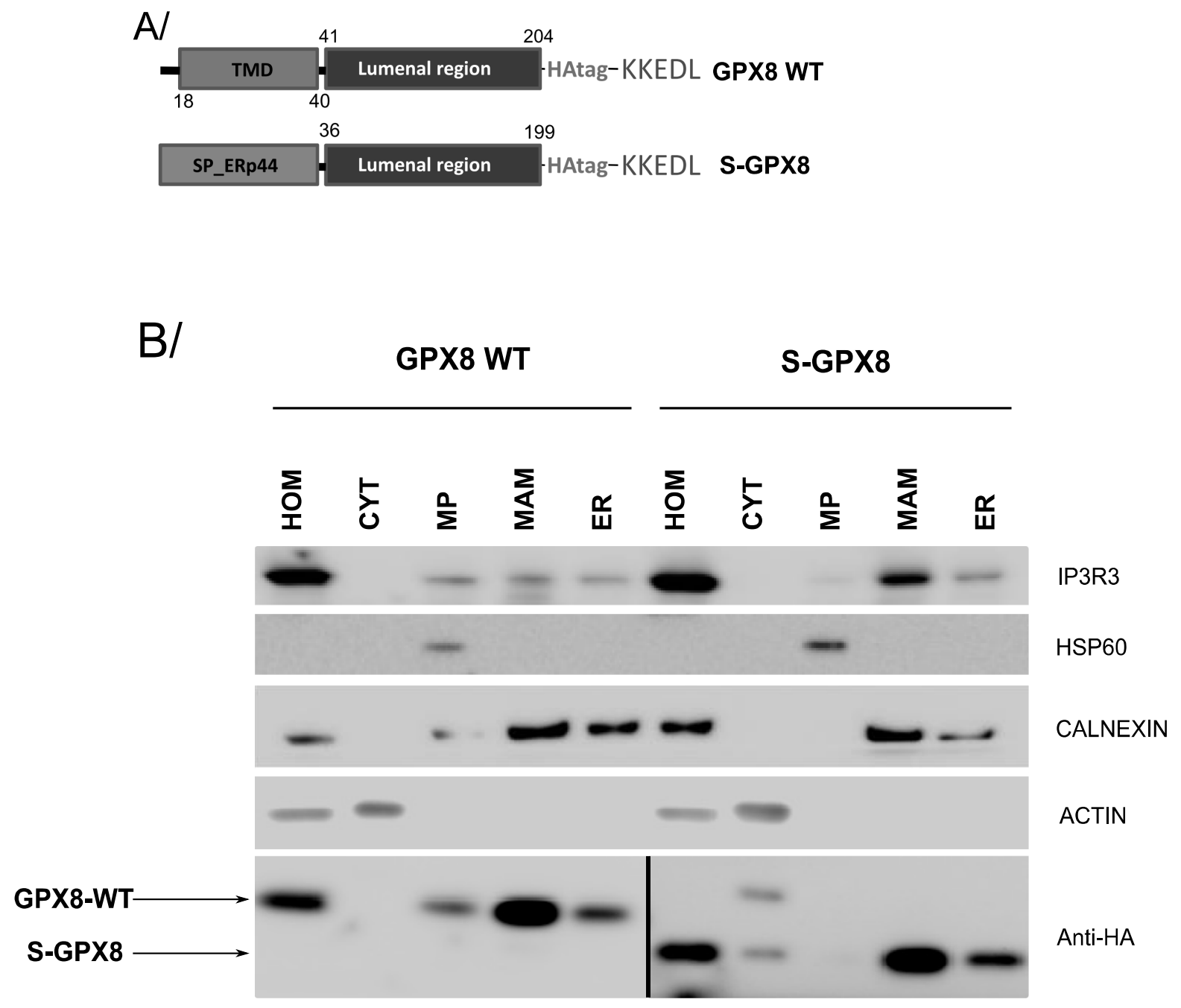




\section{Basal Mitochondrial Ca2+ levels}
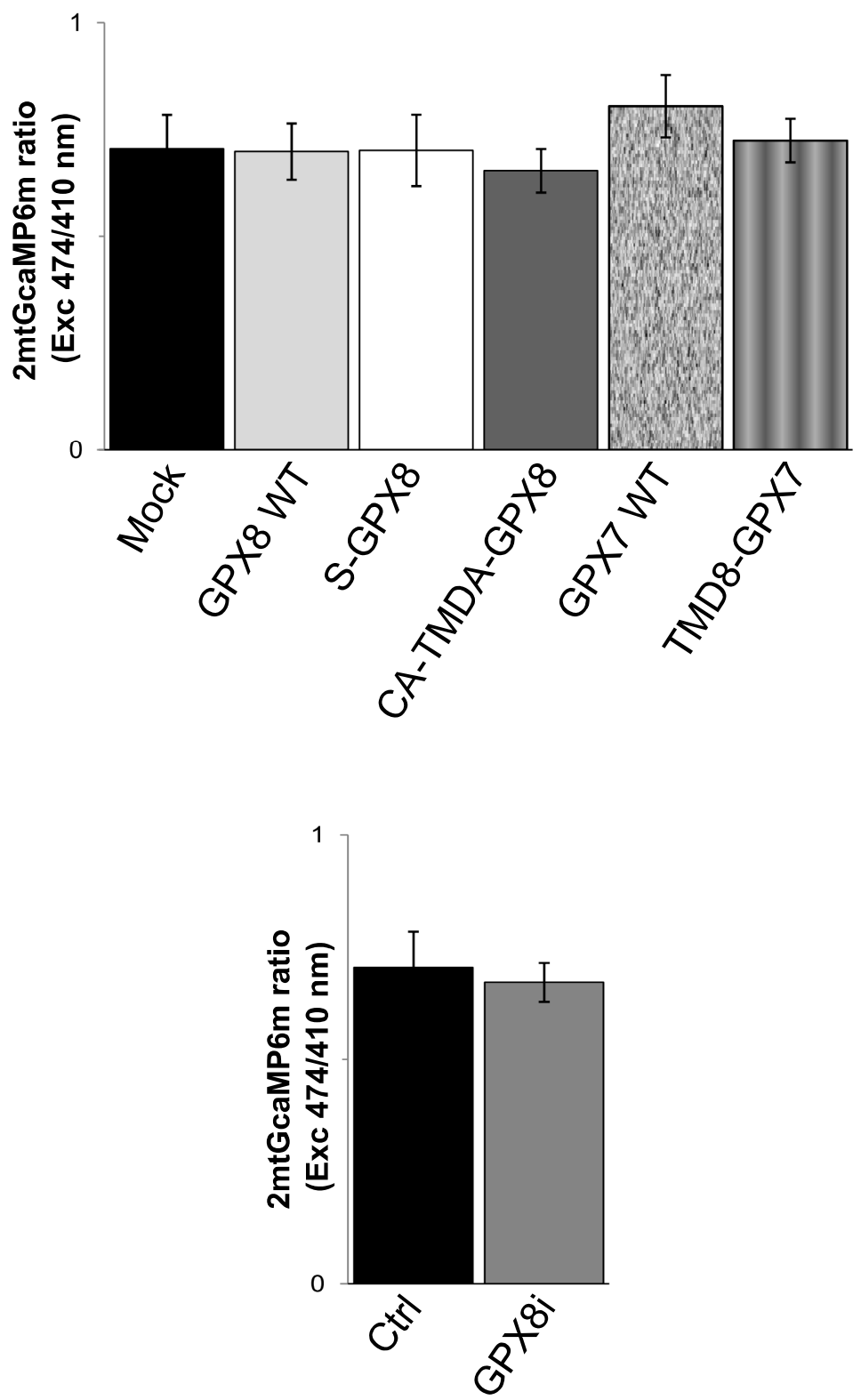
Supplementary figure S7

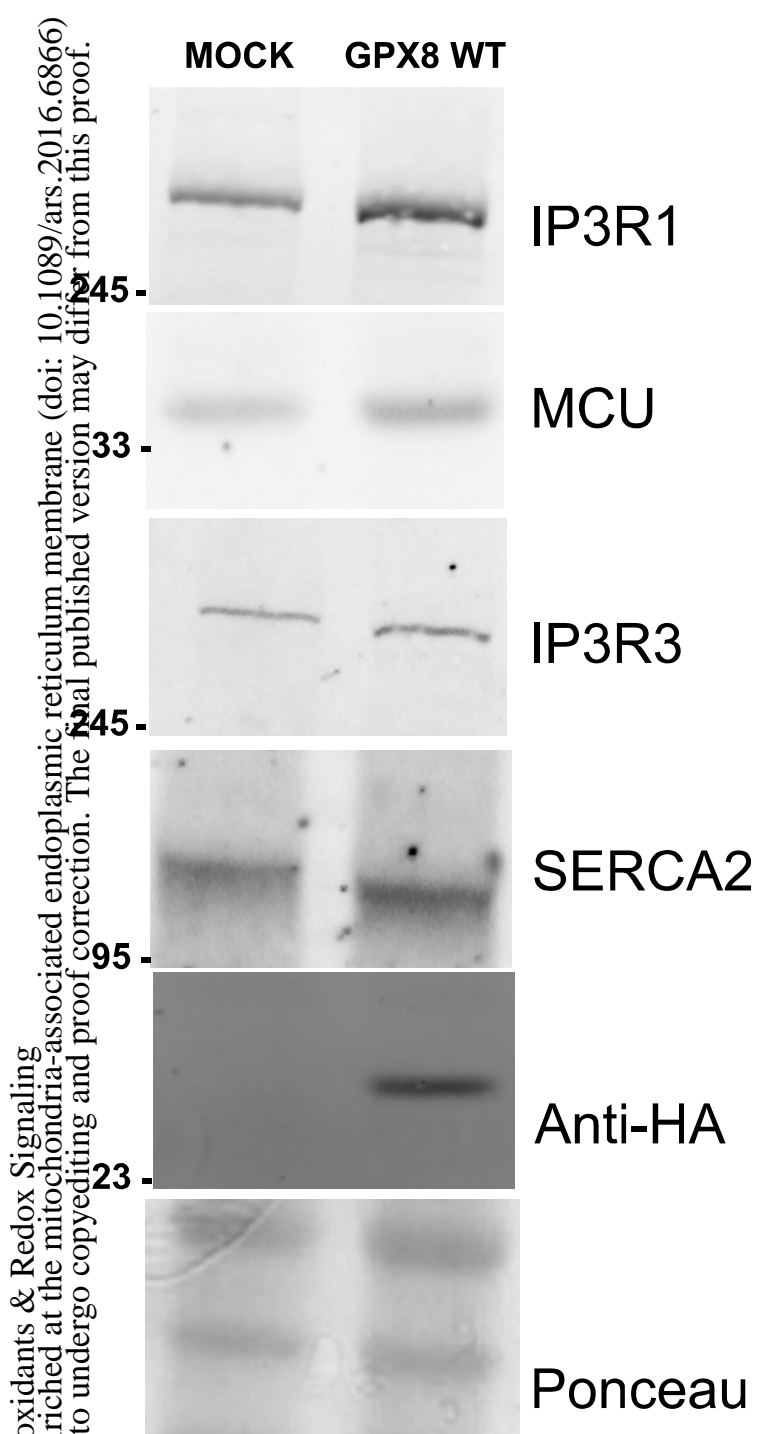




\section{Supplementary figure S8}

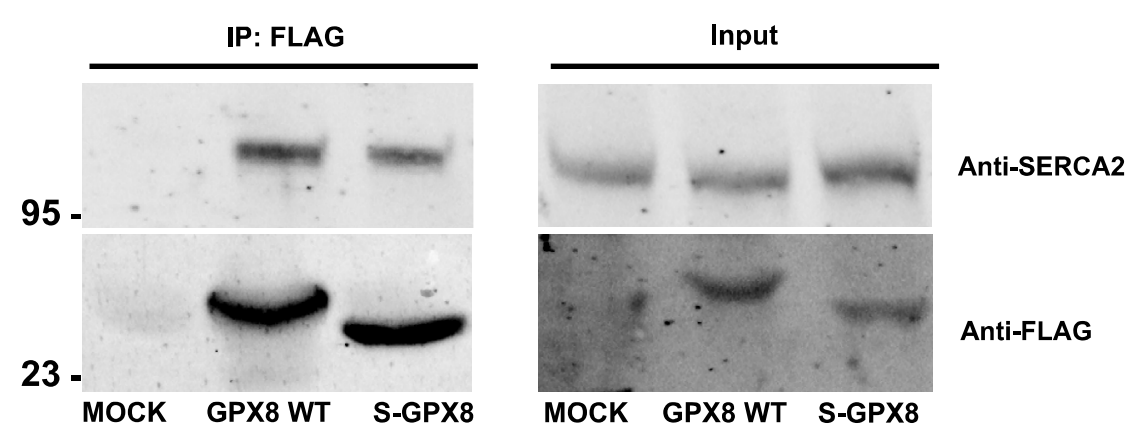


Supplementary figure S9

Western blots figure 1

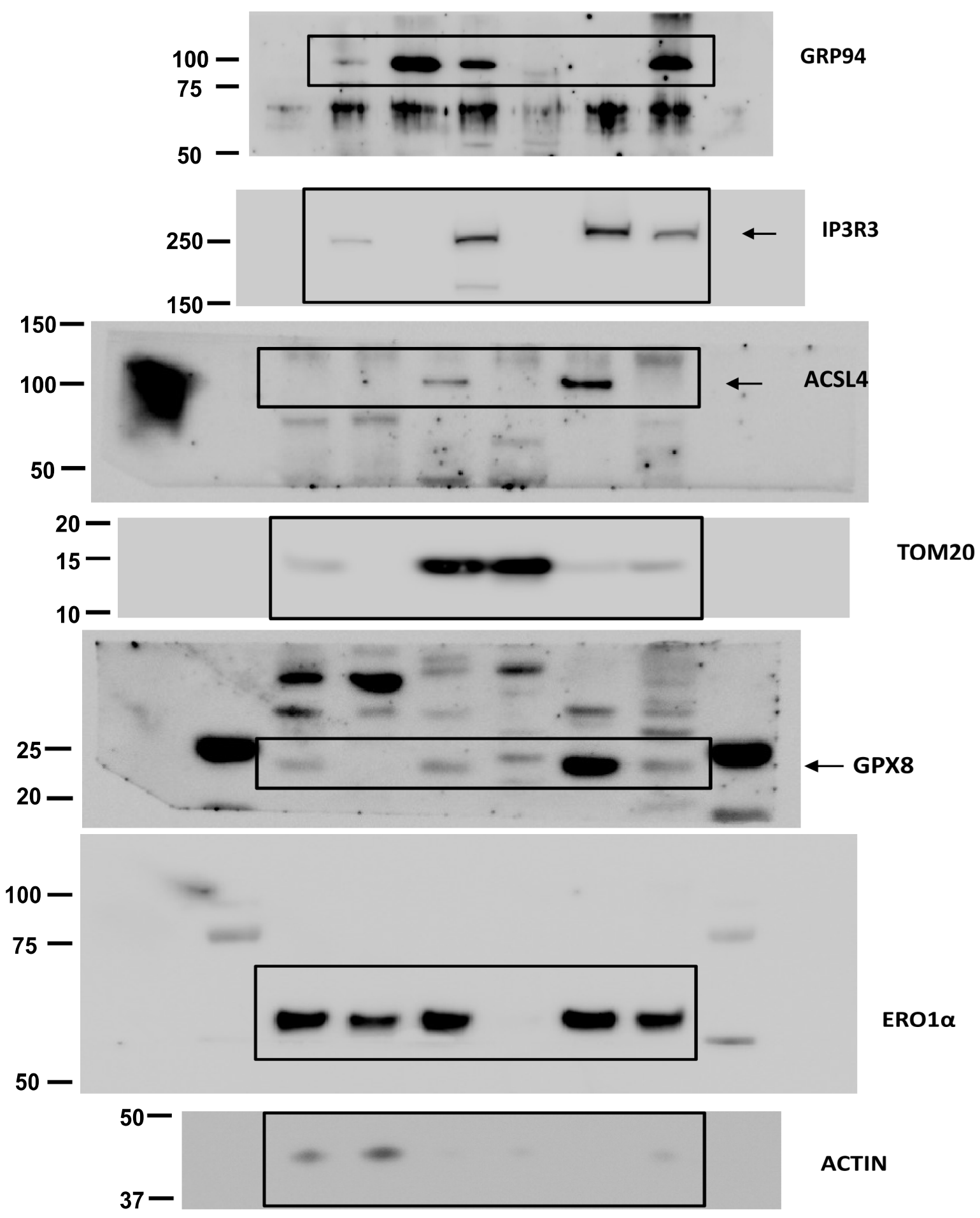




\section{Supplementary figure S10}

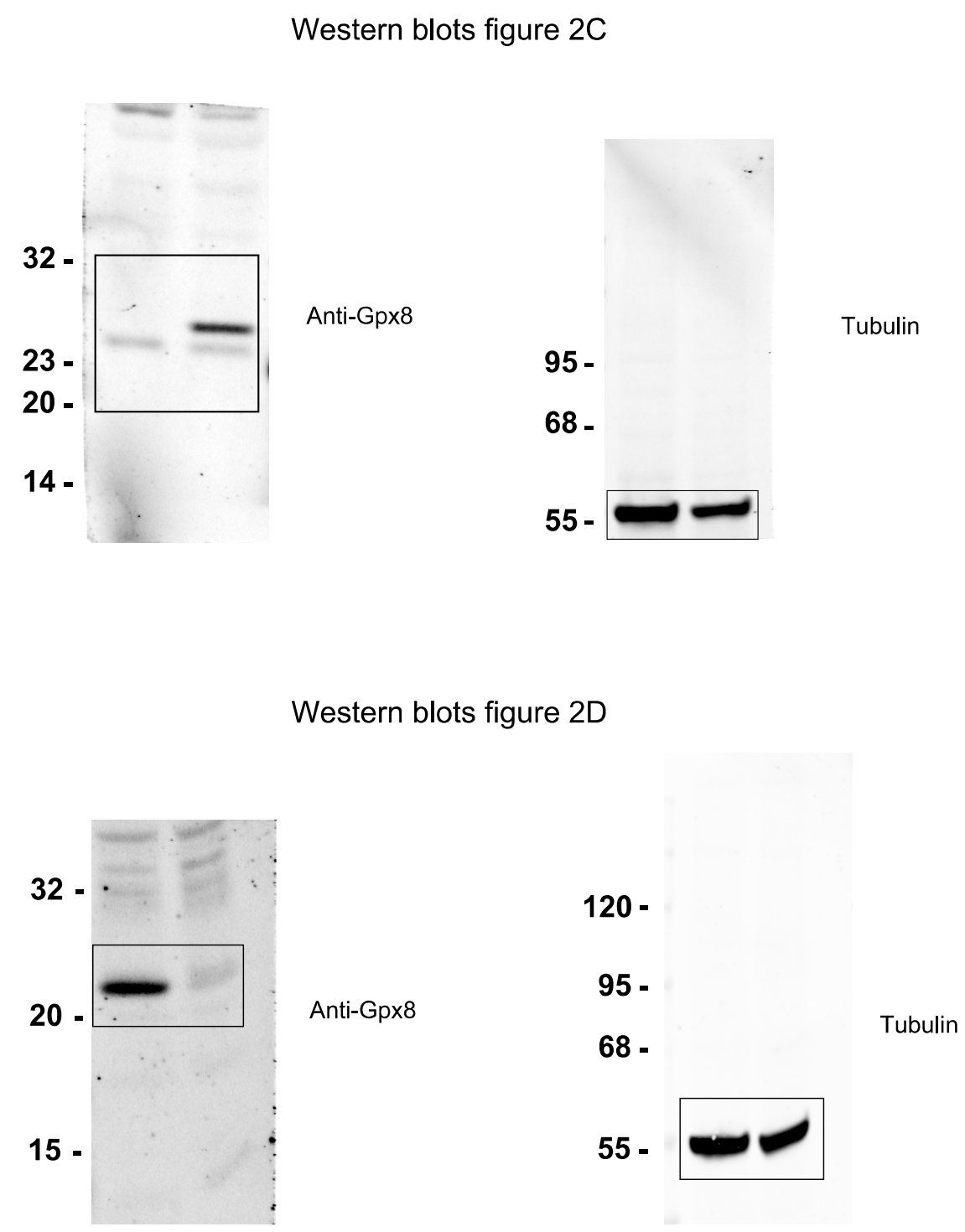




\section{Supplementary figure S11}

Western blot figure 4B

68 -

Anti-HA

55 -

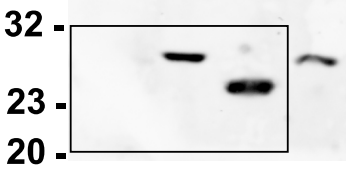




\section{Supplementary figure S12}

Western blots figure 6B

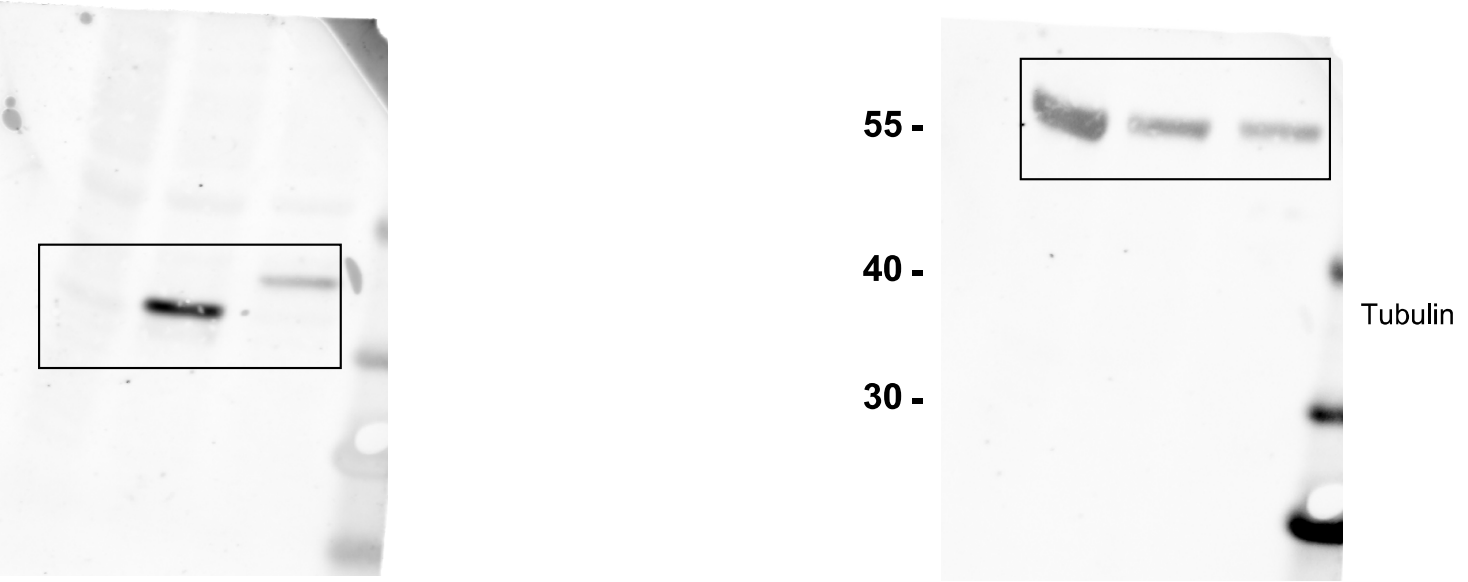

Western blots figure 6F

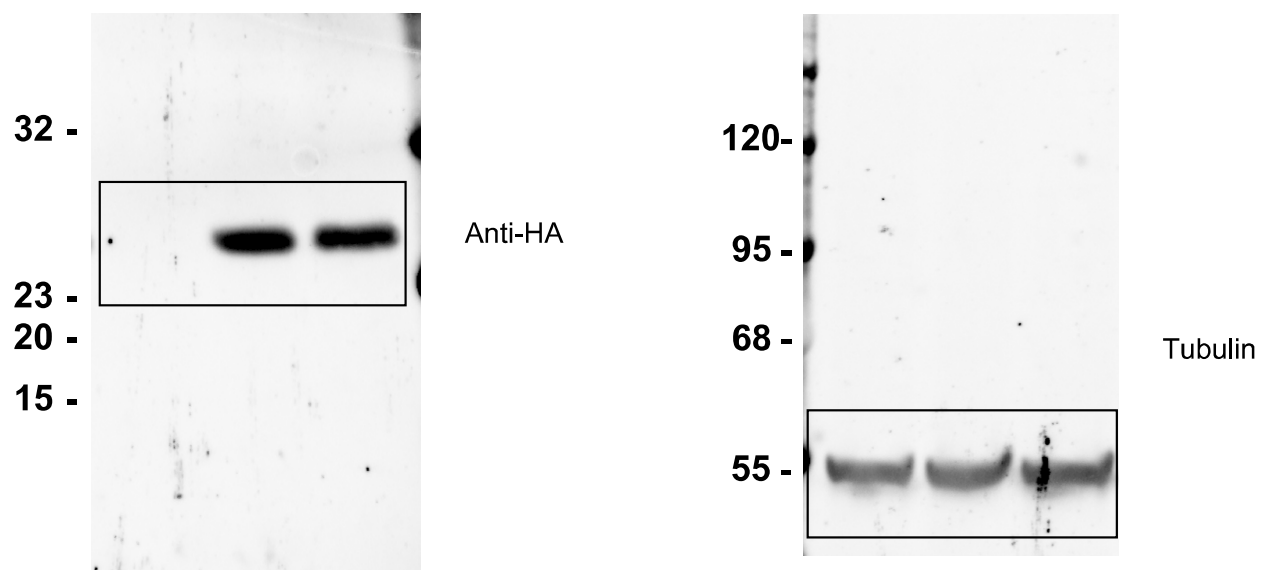




\section{Supplementary figure S13}

\section{Western blots figure 7B}

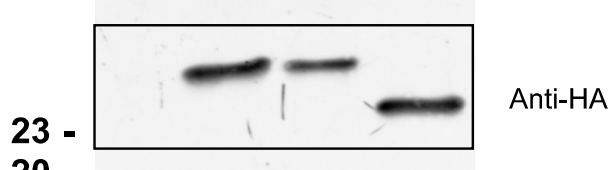

20 -

15 -

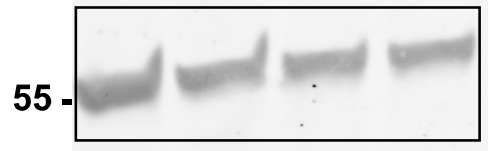

Tubulin

40 - 


\section{Supplementary figure S14}

Western blot figure 8B

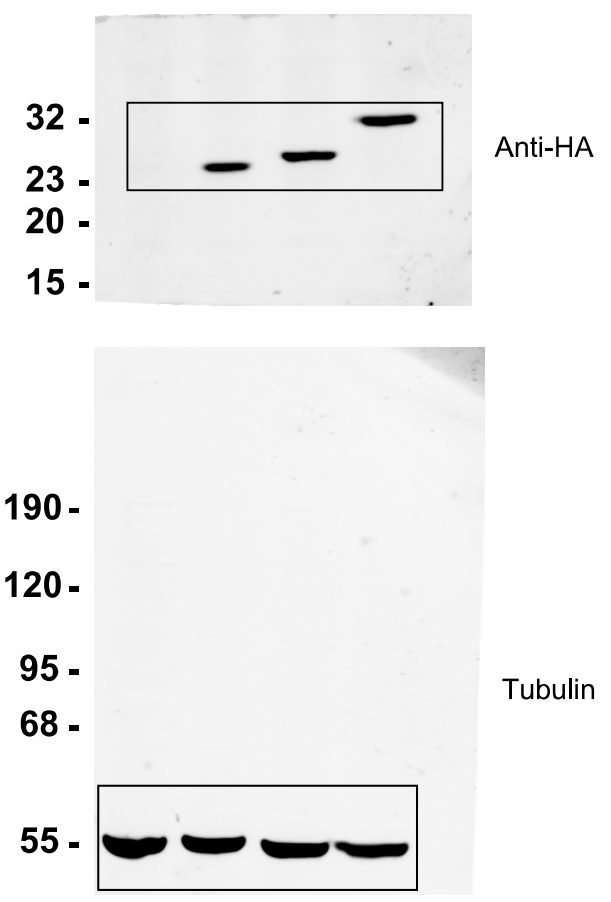




\section{Supplementary figure S15}

Western blots supplementary figure S5

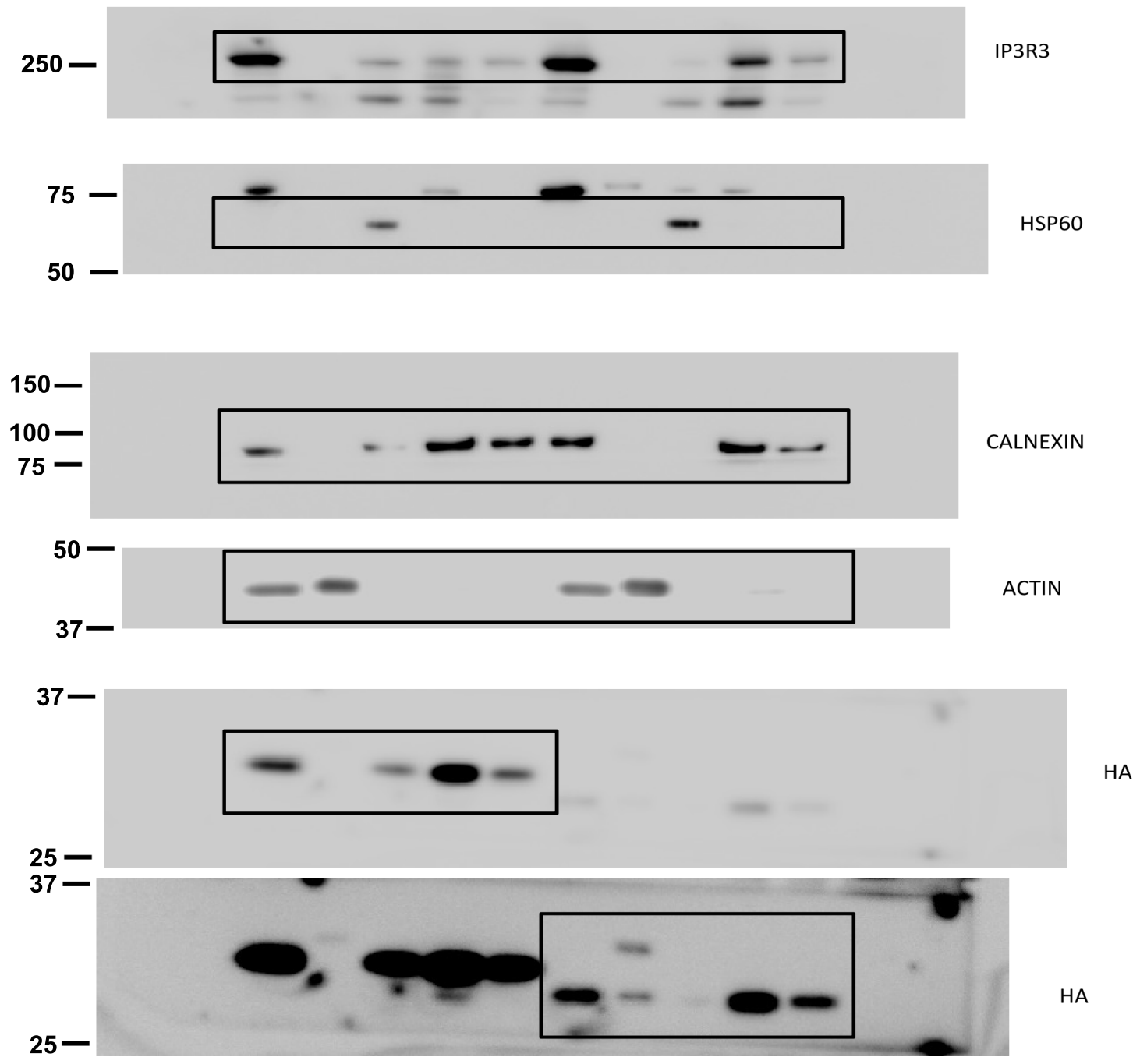




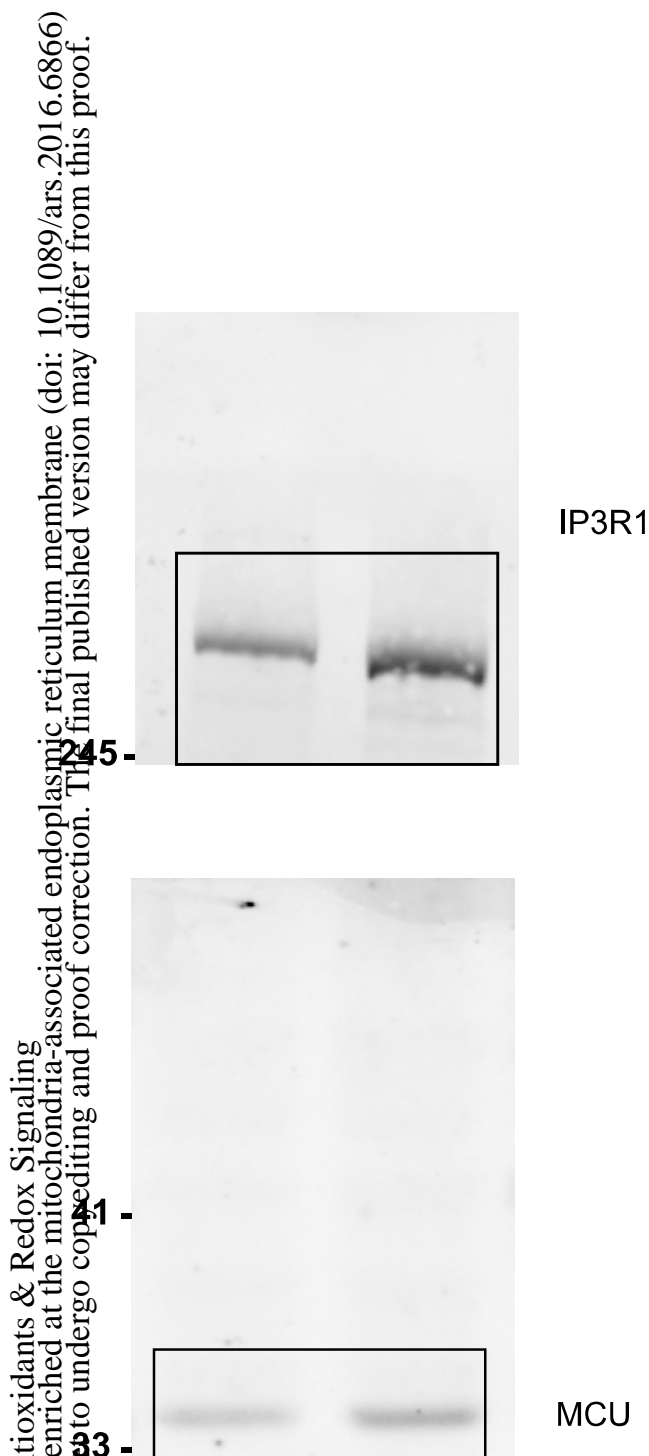

\section{Supplementary figure S16}

Western blots supplementary figure S7

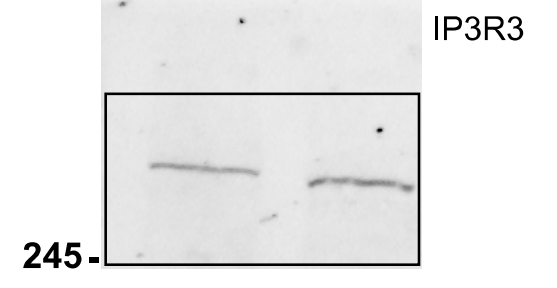

33 -

Anti-HA

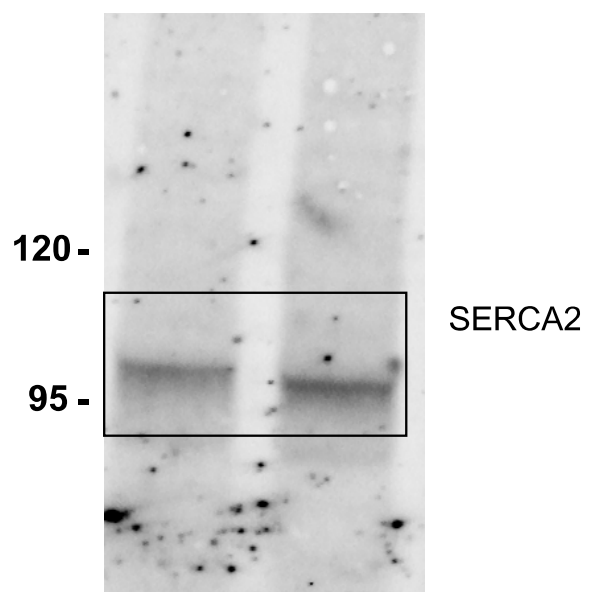

23 -

0

MCU

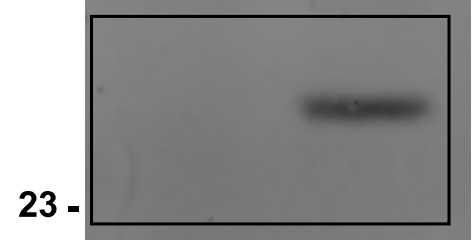

